\title{
A VARIATIONAL APPROACH TO THE MACROSCOPIC ELECTRODYNAMICS OF ANISOTROPIC HARD SUPERCONDUCTORS
}

\author{
GRAZIANO CRASTA AND ANNALISA MALUSA
}

\begin{abstract}
We consider the Bean's critical state model for anisotropic superconductors. A variational problem solved by the quasi-static evolution of the internal magnetic field is obtained as the $\Gamma$-limit of functionals arising from the Maxwell's equations combined with a power law for the dissipation. Moreover, the quasi-static approximation of the internal electric field is recovered, using a first order necessary condition.

If the sample is a long cylinder subjected to an axial uniform external field, the macroscopic electrodynamics is explicitly determined.
\end{abstract}

\section{INTRODUCTION}

It is well known that a superconductor is a conductor which is able to pass an electric current without dissipation. The transition from the normally conducting state to the superconducting one occurs at a critical temperature $T_{c}$, depending on the material, below which the material exhibits (almost) perfect conductivity. We are interested in the response of a superconducting material to an applied external magnetic field $\vec{H}_{s}$ under isothermal conditions below its critical temperature.

The superconductors can be classified in terms of a material parameter $\kappa>0$, known as the Ginzburg-Landau parameter. For the so called type-II superconductors (corresponding to $\kappa>1 / \sqrt{2}$ ) there exist two critical magnetic field intensities, $H_{c_{1}}<H_{c_{2}}$, such that for $\left|\vec{H}_{s}\right|<H_{c_{1}}$ the material is in the superconducting state and the magnetic field is excluded from the bulk of the sample except in thin boundary layers, while for $\left|\vec{H}_{s}\right|>H_{c_{2}}$ the material behaves as a normal conductor, and the magnetic field penetrates it fully. For $H_{c_{1}}<\left|\vec{H}_{s}\right|<H_{c_{2}}$ a third state exists, known as "mixed state" as well as "vortex state". The mixed state is characterized by a partial penetration of the magnetic field into the sample, which occurs, at a mesoscopic level, by means of thin filaments of normally conducting material carrying magnetic flux and circled by a vortex of superconducting current. Type-I superconductors are those with $H_{c_{1}}=H_{c_{2}}$, so that the mixed state does not occur.

Although from a physical point of view the most interesting description of the mixed state for type-II superconductors is given by the mesoscopic Ginzburg-Landau model, in designing magnets and other large-scale applications of superconducting materials, engineers use macroscopic models, involving averaged variables. One of the most reliable macroscopic models is the Bean's critical state model (see [7]). We refer to [13] for a

Date: December 20, 2006.

2000 Mathematics Subject Classification. Primary 35C15; Secondary 49J30, 49J45, 49K20.

Key words and phrases. Minimum problems with constraints, Euler equation, hard superconductors, Bean's model. 
derivation of this model as a macroscopic version of the Ginzburg-Landau model under suitable assumptions.

The basic idea of the Bean's phenomenological model for isotropic materials is that, because of physical limitations imposed by the material properties, the current density $|\vec{J}|$ cannot exceed a critical value $J_{c}$ without destroying the superconducting phase.

Moreover it is assumed that any electromotive force due to external field variations induces the maximum current density flow, according to the most effective way of shielding field variations. Hence the current density $|\vec{J}|$ is forced to be $J_{c}$ in the part of the sample where the field is penetrated, while $\vec{J}=0$ in the remaining part of the sample.

At a mesoscopic level, the critical current density $J_{c}$ corresponds to the balance between a repulsive vortex-vortex interaction and attractive forces towards the pinning centers. At a macroscopic level, the electric field is zero when $|\vec{J}|<J_{c}$ and abruptly rises to arbitrarily large values if $J_{c}$ is overrun.

Since for isotropic materials it is well-established that the electric field and the current have the same direction (at least for slowly varying external fields), in the original Bean's model the Ohm's law is replaced by a vertical current-voltage law

$$
\vec{E} \| \vec{J}, \quad|\vec{J}| \leq J_{c}, \quad \vec{E}=0 \text { if }|\vec{J}|<J_{c},
$$

which can be interpreted as the limit for $p \rightarrow+\infty$ in the power-law

$$
\vec{E} \| \vec{J}, \quad|\vec{E}|=e_{c}\left(\frac{|\vec{J}|}{\left|\overrightarrow{J_{c}}\right|}\right)^{p}
$$

(see, e.g., [10, 6, 2]). Notice that the direction of the electric field is obtained by exploiting the isotropy of the material and cannot be obtained as a consequence of the power law approximation, which involves only the intensities of the fields. Hence this approach is not appropriate to deal with anisotropic materials (see anyhow [3] for some explicit computation of the electric field in the case of infinite slab geometry, and [8] in the case of a cylindrical body with elliptic section). On the other hand, many type-II superconducting materials are anisotropic. Moreover, even if the material is isotropic, the presence of fluxflow Hall effects has to be described in terms of an anisotropic resistivity (see, e.g., [10]).

Concerning the constraints on the current, in the anisotropic case the Bean's law dictates that there exists a compact set $\Delta$ containing the origin as an interior point, such that $\vec{J}$ cannot lie outside $\Delta$ without destroying the superconducting state. Moreover $\vec{J} \in \partial \Delta$ in the penetrated region, and $\vec{J}=0$ elsewhere.

Section 3 of this paper is devoted to the description of the Bean's law as a limit of a power-like law fulfilled by the dissipation. This approach allows us to determine a priori the direction of the electric field in terms of the direction of the current for anisotropic materials.

In Section $[$ we deal with a variational statement of the critical state proposed in [1], which takes the form of a quasistatic evolution of the penetrated magnetic field, obtained combining the finite-difference expression of Faraday's law with the Bean's law. We shall give a mathematical justification of the variational model as a limiting case of the power law model for dissipation. The result is proposed in terms of $\Gamma$-convergence of functionals, which is nowadays a classical tool in the mathematical methods for the material science (see, e.g., [9, 16] and the references therein).

In the last part of the paper we focus our attention to the special case of a long cylindrical anisotropic superconductor placed into a nonstationary, uniform axial magnetic field. The 
parallel geometry enables us to make a two dimensional reduction of the problem, which can then be explicitly solved.

The plan of this part of the paper is the following. In Section 5 we describe the two dimensional reduction of the problem in the case of parallel geometry. In Section 6 we introduce some technical tool needed for the analytical description of the fields. In Section 0 we find explicitly the solution to a general class of minimum problems with a gradient constraint and we determine the Euler equation solved by the optimal function coupled with its dual function. Moreover we find the explicit form of the dual function. In Section 8 the previous results are applied to the variational model for the critical state, and we find the quasistatic evolution of both the magnetic field and the dissipation inside the superconductor. Finally, a passage to the limit on the time layer gives the explicit form of the macroscopic electrodynamics.

For what concerns the magnetic field, our result generalizes the one, valid for isotropic materials, obtained by Barrett and Prigozhin in [6] with a different method based on a evolutionary variational inequality. Due to the fact that the magnetic field is explicitly known, we can compute the full penetration time in the case of monotonic external fields as well as we can depict the well known hysteresis phenomenon. On the other hand, the knowledge of the electric field allows us to give a detailed description of the evolution of the dissipation for cylinders of anisotropic materials with a general geometry of the cross section (see Figures 11 and 2).

\section{Notation AND PRELIMINARIES}

For $\xi \in \mathbb{R}^{N},|\xi|$ will be the Euclidean norm, and $\left\langle\xi, \xi^{\prime}\right\rangle$ will denote the scalar product with $\xi^{\prime} \in \mathbb{R}^{N}$. The symbol $\times$ will be used for the cross product of vectors in $\mathbb{R}^{3}$. Given $a, b \in \mathbb{R}, a \vee b$ and $a \wedge b$ will denote respectively the maximum and the minimum of $a$ and $b$.

Given $A \subset \mathbb{R}^{N}$, we shall denote by $\operatorname{Lip}(A), C(A), C_{b}(A)$ and $C^{k}(A), k \in \mathbb{N}$ the set of functions $u: A \rightarrow \mathbb{R}$ that are respectively Lipschitz continuous, continuous, bounded and continuous, and $k$-times continuously differentiable in $A$. Moreover, $C^{\infty}(A)$ will denote the set of functions of class $C^{k}(A)$ for every $k \in \mathbb{N}$, while $C^{k, \alpha}(A)$ will be the set of functions of class $C^{k}(A)$ with Hölder continuous $k$-th partial derivatives with exponent $\alpha \in[0,1]$. Finally $L^{p}(A), W^{1, p}(A), W_{0}^{1, p}(A)$, and $L^{p}\left(A, \mathbb{R}^{d}\right), W^{1, p}\left(A, \mathbb{R}^{d}\right), W_{0}^{1, p}\left(A, \mathbb{R}^{d}\right), d>1$, will be the usual Lebesgue and Sobolev spaces of scalar or vectorial functions respectively.

Let $X$ be subset of $\mathbb{R}^{N}, N \geq 2$. We shall denote by $\partial X$ its boundary, by int $X$ its interior, and by $\bar{X}$ its closure. The characteristic function of $X$ will be denoted by $\chi_{X}$. The set $X$ is said to be of class $C^{k}, k \in \mathbb{N}$, if for every point $x_{0} \in \partial X$ there exists a ball $B=B_{r}\left(x_{0}\right)$ and a one-to-one mapping $\psi: B \rightarrow D$ such that $\psi \in C^{k}(B), \psi^{-1} \in C^{k}(D)$, $\psi(B \cap X) \subseteq\left\{x \in \mathbb{R}^{n} ; x_{n}>0\right\}, \psi(B \cap \partial X) \subseteq\left\{x \in \mathbb{R}^{n} ; x_{n}=0\right\}$. If the maps $\psi$ and $\psi^{-1}$ are of class $C^{\infty}$ or $C^{k, \alpha}(k \in \mathbb{N}, \alpha \in[0,1])$, then $X$ is said to be of class $C^{\infty}$ or $C^{k, \alpha}$ respectively.

Let $D \subset \mathbb{R}^{N}$ be a compact convex set containing 0 as an interior point and with boundary of class $C^{2}$. The gauge function $\rho_{D}$ of the set $D$ is the convex, positively 1-homogeneous function defined by

$$
\rho_{D}(\xi)=\inf \{t \geq 0 ; \xi \in t D\}, \quad \xi \in \mathbb{R}^{N} .
$$


Since $D$ is a compact set containing a neighborhood of 0 , there exist two positive constants $c_{1}<c_{2}$ such that

$$
c_{1}|\xi| \leq \rho_{D}(\xi) \leq c_{2}|\xi|, \quad \forall \xi \in \mathbb{R}^{N} .
$$

The indicator function of the set $D$ is defined by

$$
I_{D}(\xi)= \begin{cases}0 & \text { if } \xi \in D \\ +\infty & \text { if } \xi \notin D\end{cases}
$$

Since $D$ has a smooth boundary, the subgradient of the indicator function can be explicitly computed, and

$$
\partial I_{D}(\xi)= \begin{cases}\left\{\alpha D \rho_{D}(\xi): \alpha \geq 0\right\} & \text { if } \xi \in \partial D \\ \emptyset & \text { if } \xi \notin D \\ \{0\} & \text { if } \xi \in \operatorname{int} D\end{cases}
$$

(see e.g. [22], Section 23).

In what follows, a family of objects, even if parameterized by a continuous parameter, will also be often called a "sequence" not to overburden notation.

\section{The PHYSICAL SETTING}

Since we shall deal with a macroscopic model, the coarse-grained electrodynamics will be formulated in terms of

(i) the flux density $\vec{B}$ within the sample, which is the average of the microscopic field intensity;

(ii) the magnetic field $\vec{H}$, which is assumed to be linearly connected to $\vec{B}$ by $\vec{B}=\mu_{0} \vec{H}$;

(iii) the averaged current density $\vec{J}$, linked to $\vec{H}$ by the Ampère's law $\vec{J}=\operatorname{curl} \vec{H}$. Moreover, on neglecting finite size effects, we can assume that

(iv) the magnetic source $\vec{H}_{s}$ enters as a boundary condition for the flux density at the surface of the sample, requiring that $\vec{n} \times \vec{H}=\vec{n} \times \vec{H}_{s}$ on that surface, where $\vec{n}$ is the outward normal vector.

Time variations of the external field $\vec{H}_{s}$ induce in the sample an electric field $\vec{E}$, according to Faraday's law. On the other hand, the electric field leads a current $\vec{J}$ which induces an internal magnetic field $\vec{H}$, according to Ampère's law. Finally, we recall that $\operatorname{div} \vec{H}=0$ (Gauss' law).

Summarizing, we are considering the so-called eddy current model for Maxwell equations:

$$
\begin{cases}\operatorname{curl} \vec{E}+\mu_{0} \frac{\partial \vec{H}}{\partial t}=0 & \text { (Faraday's law), } \\ \vec{J}=\operatorname{curl} \vec{H} & \text { (Ampère's law) } \\ \operatorname{div} \vec{H}=0 & \text { (Gauss' law), } \\ \vec{n} \times\left(\vec{H}-\vec{H}_{s}\right)=0 & \text { on the surface. }\end{cases}
$$

In order to complete the physical setting, it remains to find an appropriate version of the constitutive law $\vec{E}(\vec{J})$. Our starting point is a reading of the macroscopic behaviour of the material in terms of the dissipation $\dot{S}=\langle\vec{E}(\vec{J}), \vec{J}\rangle$ (here $S$ denotes the entropy of the system). Namely, the Bean's model dictates that if $\vec{J}$ is in the interior of an allowed region $\Delta$, which is assumed to be a convex compact set of $\mathbb{R}^{3}$ having the origin as an interior 
point, then $\dot{S}$ vanishes as no electric field is generated in stationary condition, while when $\vec{J}$ touches the boundary $\partial \Delta$ of the allowed region a huge dissipation occurs, destroying the superconducting phase. The fact that $\dot{S}=0$ if $\vec{J}$ is an interior point of $\Delta$, while $\dot{S}=+\infty$ if $\vec{J} \notin \Delta$ suggests that the Bean's law can be interpreted as the limit as $p \rightarrow \infty$ of a power law for the dissipation $\left\langle\vec{E}_{p}(\vec{J}), \vec{J}\right\rangle$, that is

$$
\left\langle\vec{E}_{p}(\vec{J}), \vec{J}\right\rangle=\frac{c}{p}\left(\rho_{\Delta}(\vec{J})\right)^{p} .
$$

This approximation allows us to recover the direction of the electric field $\vec{E}(\vec{J})$ when $\vec{J} \in \partial \Delta$. Namely, we claim that, under the physically consistent assumption that

$$
\lim _{t \rightarrow 0^{+}} \vec{E}_{p}(t \vec{J})=0, \quad \forall \vec{J} \in \mathbb{R}^{3},
$$

the relation (5) implies, for $p>1$,

$$
\vec{E}_{p}(\vec{J})=\frac{c}{p}\left(\rho_{\Delta}(\vec{J})\right)^{p-1} D \rho_{\Delta}(\vec{J}) .
$$

In order to prove (7), we differentiate (5), obtaining

$$
\left\langle D \vec{E}_{p}(\vec{J}), \vec{J}\right\rangle+\vec{E}_{p}(\vec{J})=c\left(\rho_{\Delta}(\vec{J})\right)^{p-1} D \rho_{\Delta}(\vec{J}) .
$$

Hence, fixed $\vec{J} \neq 0$, the function $v(t)=\vec{E}_{p}(t \vec{J}), t>0$, is a solution of the O.D.E.

$$
\left\{\begin{array}{l}
t v^{\prime}(t)+v(t)=c t^{p-1} w \\
v(1)=\vec{E}_{p}(\vec{J})
\end{array}\right.
$$

where $w=\left(\rho_{\Delta}(\vec{J})\right)^{p-1} D \rho_{\Delta}(\vec{J})$. Then

$$
v(t)=\frac{1}{t}\left[\vec{E}_{p}(\vec{J})+\frac{c w}{p}\left(t^{p}-1\right)\right]
$$

and, by (6),

$$
\vec{E}_{p}(\vec{J})-\frac{c}{p}\left(\rho_{\Delta}(\vec{J})\right)^{p-1} D \rho_{\Delta}(\vec{J})=\lim _{t \rightarrow 0^{+}}\left[\vec{E}_{p}(\vec{J})+\frac{c w}{p}\left(t^{p}-1\right)\right]=0 .
$$

As a consequence of $(\mathbb{J})$, the direction of $\vec{E}_{p}(\vec{J})$ is given by $D \rho_{\Delta}(\vec{J})$, and it does not depend on $p$. In conclusion, recalling (4), the anisotropic version of the Bean's law is the following.

(B1) There exists a convex compact set $\Delta$ containing the origin as an interior point and such that $\vec{J}(x, t) \in \Delta$ for every $x \in \Omega, t \geq 0$.

(B2) If we denote by $I_{\Delta}$ the indicator function of the set $\Delta$, the constitutive law $\vec{E}(\vec{J})$ is given by $\vec{E} \in \partial I_{\Delta}(\vec{J})$.

\section{A variational model for the mixed state}

Let $A \subset \mathbb{R}^{3}$ be the region occupied by the superconductor, and $\partial A$ its surface. We assume that $A$ is a bounded, open subset of $\mathbb{R}^{3}$, and that $\partial A$ is of class $C^{1,1}$. Moreover, we assume that $A$ has no enclosed cavity. In our model the presence of cavities is not allowed, since the boundary condition in such a cavity cannot be given in terms of the external magnetic field. (The magnetic field must be constant on the boundary of every enclosed cavity.) In order to avoid the presence of cavities, we assume that $A$ has second Betti number 0 (see e.g. [19] for the definition of Betti numbers). 
Let $T>0$ be fixed. For every $n \in \mathbb{N}^{+}$, we take a partition $P^{n}=\left\{t_{i}^{n}\right\}_{i=0}^{k(n)}$ of the interval $[0, T]$, and we set $\delta t_{i}^{n}=t_{i}^{n}-t_{i-1}^{n}$. The least action principle proposed in [1] is the following: starting from an initial field profile $\vec{H}_{i}^{n}(x)$ in $A$ at the time layer $i$, and under a small change of the external drive, the new profile $\vec{H}_{i+1}^{n}(x)$ at the time layer $i+1$ is the unique solution to the minimum problem

$$
\min \left\{\int_{A}\left|\vec{V}-\vec{H}_{i}^{n}\right|^{2} d x ; \vec{V} \in \vec{H}_{s}\left(t_{i+1}^{n}\right)+X_{2}, \operatorname{curl} \vec{V} \in \Delta \text { a.e. }\right\},
$$

where, for every $p>1$,

$$
X_{p}=\left\{\vec{V} \in L^{p}\left(A, \mathbb{R}^{3}\right) ; \operatorname{curl} \vec{V} \in L^{p}\left(A, \mathbb{R}^{3}\right), \operatorname{div} \vec{V}=0, \vec{n} \times \vec{V}=0 \text { on } \partial A\right\} .
$$

In [1] the trustworthiness of this variational principle was motivated in terms of nonequilibrium thermodynamical principles in analogy with the ohmic case. In this section we shall give a mathematical justification, in terms of $\Gamma$-convergence of functionals, of the variational model as a limiting case of the power law model for dissipation.

Our starting point is the fact that, assuming that the power law (5) holds true, the discretized version of the Faraday's law

$$
\operatorname{curl} \vec{E}_{i+1}^{n}+\frac{\mu_{0}}{c \delta t_{i}^{n}}\left(\vec{H}_{i+1}^{n}-\vec{H}_{i}^{n}\right)=0
$$

is the Euler equation for the functional

$$
F_{p}(\vec{V})=\int_{A} \frac{1}{p} \rho_{\Delta}(\operatorname{curl} \vec{V})^{p}+\lambda\left|\vec{V}-\vec{H}_{i}^{n}\right|^{2} d x, \quad \vec{V} \in \vec{H}_{s}\left(t_{i+1}^{n}\right)+X_{p}
$$

where $\lambda:=\frac{\mu_{0}}{2 c \delta t_{i}^{n}}>0$.

In other words, it can be easily checked that the magnetic field $\vec{H}_{i+1}^{n}$ is the unique minimum point of $F_{p}$ in $\vec{H}_{s}\left(t_{i+1}^{n}\right)+X_{p}$. It is clear that, up to a translation of a constant vector, it is equivalent to minimize $F_{p}$ on $X_{p}$ by changing $\vec{H}_{i}^{n}$ with $\vec{H}_{i}^{n}-\vec{H}_{s}\left(t_{i+1}^{n}\right)$.

The following properties of the spaces $X_{p}$ will be useful in the sequel (see [25, Thm. 2.2] and [24, Thm. 3.1]).

Theorem 4.1. Let $A \subset \mathbb{R}^{3}$ be a bounded open set of class $C^{1,1}$. Let $1<p<\infty$. Then the following hold.

(i) Every function $\vec{V} \in X_{p}$ belongs to $W^{1, p}\left(A, \mathbb{R}^{3}\right)$. Moreover, if $p>3$, then $\vec{V} \in$ $C^{0, \alpha}(\bar{A})$ and there exists a constant $C_{p}>0$, depending only on $p$ and $A$, such that

$$
\|\vec{V}\|_{C^{0, \alpha}(\bar{A})} \leq C_{p}\|\operatorname{curl} \vec{V}\|_{p} .
$$

(ii) If $A$ has second Betti number 0 , then there exists a constant $M_{p}>0$, depending only on $p$ and $A$, such that

$$
\|D \vec{V}\|_{p} \leq M_{p}\|\operatorname{curl} \vec{V}\|_{p}
$$

for every $\vec{V} \in X_{p}$.

Remark 4.2. From Theorem 4.1 we infer that $X_{p}$ is a (proper) subspace of $W^{1, p}\left(A, \mathbb{R}^{3}\right)$. Moreover, for $p>3$ the map defined by $X_{p} \ni \vec{V} \mapsto\|\operatorname{curl} \vec{V}\|_{p}$ is a norm in $X_{p}$ equivalent to the standard $W^{1, p}$ norm. 
From now on we shall always assume $p>3$. In this case, by Theorem 4.1(i), a function $\vec{V} \in X_{p}$ is continuous in $\bar{A}$, and the boundary condition $\vec{n} \times \vec{V}$ is understood to be pointwise fulfilled. On the other hand, the divergence-free requirement on $\vec{V} \in X_{p}$ is understood in the sense of distributions, i.e.

$$
\int_{A} \vec{V} \cdot D \vec{\Psi}=0, \quad \forall \vec{\Psi} \in C_{0}^{\infty}\left(A, \mathbb{R}^{3}\right) .
$$

For our subsequent considerations, we need to define all the functionals $F_{p}$ on the same Banach space. For this reason, naming $\vec{U}_{0}=\vec{H}_{i}^{n}-\vec{H}_{s}\left(t_{i+1}^{n}\right)$, we set

$$
F_{p}(\vec{V})= \begin{cases}\int_{A} \frac{1}{p} \rho_{\Delta}(\operatorname{curl} \vec{V})^{p}+\lambda\left|\vec{V}-\vec{U}_{0}\right|^{2} d x, & \text { if } \vec{V} \in X_{p}, \\ +\infty, & \text { otherwise in } L^{2}\left(A, \mathbb{R}^{3}\right) .\end{cases}
$$

We also define the functional

$$
F(\vec{V})= \begin{cases}\int_{A} I_{\Delta}(\operatorname{curl} \vec{V})+\left|\vec{V}-\vec{U}_{0}\right|^{2} d x, & \text { if } \vec{V} \in X_{2}, \\ +\infty, & \text { otherwise in } L^{2}\left(A, \mathbb{R}^{3}\right),\end{cases}
$$

where $I_{\Delta}$ is the indicator function of the convex set $\Delta$, defined in (3).

Lemma 4.3. The functionals $F_{p}, p>3$, and $F$ are lower semicontinuous in the strong $L^{2}$ topology. Moreover, the functionals $F_{p}$ are equicoercive, i.e. there exists a constant $\alpha>0$ such that

$$
F_{p}(\vec{V}) \geq \frac{\lambda}{2}\|\vec{V}\|_{2}^{2}-\alpha \quad \forall \vec{V} \in L^{2}\left(A, \mathbb{R}^{3}\right), \forall p>3 .
$$

Proof. Let $3<p<\infty$, and let $\left(\vec{V}_{k}\right) \subset L^{2}\left(A, \mathbb{R}^{3}\right)$ be a sequence converging to $\vec{V}$ in $L^{2}$. We have to prove that

$$
F_{p}(\vec{V}) \leq \liminf _{k \rightarrow \infty} F_{p}\left(\vec{V}_{k}\right) .
$$

Without loss of generality we can assume that $\vec{V}_{k} \in X_{p}$ for every $k$ and

$$
\lim _{k \rightarrow \infty} F_{p}\left(\vec{V}_{k}\right)=C<+\infty .
$$

We claim that $\vec{V} \in X_{p}$. Namely, the divergence-free requirement is stable under strong $L^{2}$ convergence. Moreover, from (12) and (2) we have that there exists a constant $C_{1}>0$ such that $\left\|\operatorname{curl} \vec{V}_{k}\right\|_{p} \leq C_{1}$ for every $k \in \mathbb{N}$. From Theorem 4.1(i) we infer that the sequence $\left(\vec{V}_{k}\right)$ is equicontinuous and equibounded in $\bar{A}$. Hence we can pass to a subsequence, that we do not relabel, converging uniformly to $\vec{V}$ in $\bar{A}$, so that the boundary condition $\vec{n} \times \vec{V}=0$ on $\partial A$ holds. Finally, from Remark 4.2, $\left(\vec{V}_{k}\right)$ converges to $\vec{V}$ weakly in $W^{1, p}\left(A, \mathbb{R}^{3}\right)$, and $\|\operatorname{curl} \vec{V}\|_{p} \leq \liminf _{k}\left\|\operatorname{curl} \vec{V}_{k}\right\|_{p}$, proving the claim.

By the convexity of $\rho_{\Delta}$ and the convergence of $\operatorname{curl} \vec{V}_{k}$ to $\operatorname{curl} \vec{V}$ in the weak $L^{p}$ topology, we obtain that

$$
\int_{A} \rho_{\Delta}(\operatorname{curl} \vec{V})^{p} \leq \liminf _{k \rightarrow \infty} \int_{A} \rho_{\Delta}\left(\operatorname{curl} \vec{V}_{k}\right)^{p},
$$

which implies the semicontinuity inequality $F_{p}(\vec{V}) \leq C$.

The semicontinuity of $F$ can be proved in a similar way, while the equicoerciveness of $\left(F_{p}\right)$ easily follows choosing $\alpha=\frac{\lambda}{2}\left\|\vec{U}_{0}\right\|_{2}^{2}$. 
Remark 4.4. For every $p>3$, the functional $F_{p}$ is lower semicontinuous in $L^{2}\left(A, \mathbb{R}^{3}\right)$, coercive, and strictly convex in its effective domain $X_{p}$. Hence it admits a unique minimizer $\vec{U}_{p}$, which belongs to $X_{p}$. Similarly, the functional $F$ admits a unique minimizer $\vec{U} \in X_{2}$ with $\rho_{\Delta}(\operatorname{curl} \vec{U}) \leq 1$.

The mathematical justification of the Badía and López model is based on the fact that the sequence $\left(\vec{U}_{p}\right)$ of minimizers of $\left(F_{p}\right)$ converges to the minimizer $\vec{U}$ of $F$. As is customary in material science, we are going to prove this statement using the technique of $\Gamma$-convergence (see [9] and the reference therein for examples of applications of the theory to different models).

Definition 4.5. The sequence $\left(F_{p}\right) \Gamma$-converges to the functional $F$ (with respect to the strong $L^{2}$ topology) if the following two conditions are satisfied.

(i) For every $\vec{V} \in L^{2}\left(A, \mathbb{R}^{3}\right)$ there exists a sequence $\left(\vec{V}_{p}\right)$ (called a recovering sequence), converging to $\vec{V}$ in $L^{2}\left(A, \mathbb{R}^{3}\right)$, and such that

$$
\limsup _{p \rightarrow \infty} F_{p}\left(\vec{V}_{p}\right) \leq F(\vec{V}) .
$$

(ii) For every sequence $\left(\vec{V}_{p}\right) \subset L^{2}\left(A, \mathbb{R}^{3}\right)$ converging strongly to $\vec{V}$, we have

$$
\liminf _{p \rightarrow \infty} F_{p}\left(\vec{V}_{p}\right) \geq F(\vec{V}) .
$$

Remark 4.6. Since the functionals $F_{p}$ are equicoercive (see Lemma 4.3), the convergence of the minimizers $\left(\vec{U}_{p}\right)$ to $\vec{U}$ will follow if we prove that $\left(F_{p}\right) \Gamma$-converges to $F$ and that $\left(\vec{U}_{p}\right)$ is a precompact sequence in $L^{2}\left(A, \mathbb{R}^{3}\right)$ (see e.g. [9]).

The main tool needed in the proof of the $\Gamma$-convergence of $\left(F_{p}\right)$ to $F$ is the following lemma.

Lemma 4.7. Let $\left(\vec{V}_{p}\right) \in L^{2}\left(A, \mathbb{R}^{3}\right)$ be a sequence converging to $\vec{V}$ in $L^{2}\left(A, \mathbb{R}^{3}\right)$. If

$$
\liminf _{p \rightarrow \infty} F_{p}\left(\vec{V}_{p}\right)<+\infty
$$

then $\rho_{\Delta}(\operatorname{curl} \vec{V}) \leq 1$ a.e. in $A$.

Proof. Without loss of generality we can assume that

$$
\begin{array}{r}
\liminf _{p \rightarrow \infty} F_{p}\left(\vec{V}_{p}\right)=\lim _{p \rightarrow \infty} F_{p}\left(\vec{V}_{p}\right)=L<+\infty, \\
\operatorname{curl} \vec{V}_{p} \in L^{p}\left(A, \mathbb{R}^{3}\right), \quad F_{p}\left(\vec{V}_{p}\right) \leq L_{1} \quad \forall p>3 .
\end{array}
$$

We are going to prove that the sequence $\left(\vec{V}_{p}\right)$ is bounded in $W^{1, q}\left(A, \mathbb{R}^{3}\right)$ for every $q>3$. In view of Theorem 4.1, it is enough show that for every given $q>3$ there exists a constant $C>0$ such that $\left\|\operatorname{curl} \vec{V}_{p}\right\|_{q} \leq C$ for every $p>q$.

Given $3<q<p$, by (2) and Hölder's inequality we have

$$
\begin{aligned}
\int_{A}\left|\operatorname{curl} \vec{V}_{p}\right|^{q} d x & \leq \frac{1}{c_{1}^{q}} \int_{A} \rho_{\Delta}\left(\operatorname{curl} \vec{V}_{p}\right)^{q} d x \\
& \leq \frac{1}{c_{1}^{q}}\left(\frac{p}{|A|}\right)^{q / p}|A|\left(\int_{A} \frac{1}{p} \rho_{\Delta}\left(\operatorname{curl} \vec{V}_{p}\right)^{p} d x\right)^{q / p} .
\end{aligned}
$$

On the other hand we get

$$
\int_{A} \frac{1}{p} \rho_{\Delta}\left(\operatorname{curl} \vec{V}_{p}\right)^{p} d x \leq F_{p}\left(\vec{V}_{p}\right) \leq L_{1} .
$$


Collecting all the previous estimates, for every $p>q$ we obtain

$$
\int_{A}\left|\operatorname{curl} \vec{V}_{p}\right|^{q} d x \leq \frac{|A|}{c_{1}^{q}}\left(\frac{p L_{1}}{|A|}\right)^{q / p} \leq \frac{|A|}{c_{1}^{q}} \exp \left(\frac{q L_{1}}{e|A|}\right) .
$$

Then the sequence $\left(\vec{V}_{p}\right)$ is bounded in $W^{1, q}\left(A, \mathbb{R}^{3}\right)$, and hence $\left(\vec{V}_{p}\right)$ converges to $\vec{V}$ in the weak topology of $W^{1, q}\left(A, \mathbb{R}^{3}\right)$.

This fact and the convexity of the function $\rho_{\Delta}$ imply that

$$
\int_{B} \rho_{\Delta}(\operatorname{curl} \vec{V}) d x \leq \liminf _{p \rightarrow \infty} \int_{B} \rho_{\Delta}\left(\operatorname{curl} \vec{V}_{p}\right) d x
$$

for every open set $B \subseteq A$ (see e.g. [11, Thm. 4.2.1]). On the other hand, by Hölder's inequality

$$
\begin{aligned}
\int_{B} \rho_{\Delta}\left(\operatorname{curl} \vec{V}_{p}\right) d x & \leq\left(\int_{B} \rho_{\Delta}\left(\operatorname{curl} \vec{V}_{p}\right)^{p} d x\right)^{1 / p}|B|^{(p-1) / p} \\
& \leq p^{1 / p}\left(\frac{1}{p} \int_{B} \rho_{\Delta}\left(\operatorname{curl} \vec{V}_{p}\right)^{p} d x\right)^{1 / p}|B|^{(p-1) / p} .
\end{aligned}
$$

Then, using (14) and (15) we get $\int_{B} \rho_{\Delta}(\operatorname{curl} \vec{V}) d x \leq|B|$. Since $\rho_{\Delta}(\operatorname{curl} \vec{V}) \in L^{1}(A)$, we conclude that

$$
\rho_{\Delta}(\operatorname{curl} \vec{V}(x))=\lim _{r \rightarrow 0^{+}} \frac{1}{\left|B_{r}(x)\right|} \int_{B_{r}(x)} \rho_{\Delta}(\operatorname{curl} \vec{V}) d x \leq 1,
$$

for a.e. $x \in A$.

Theorem 4.8. The sequence $\left(F_{p}\right) \Gamma$-converges to $F$.

Proof. (i) Let $\vec{V} \in L^{2}\left(A, \mathbb{R}^{3}\right)$. It is not restrictive to assume that $F(\vec{V})<+\infty$, so that $\vec{V} \in X_{2}$ and $\rho_{\Delta}(\operatorname{curl} \vec{V}) \leq 1$ a.e. Then it is enough to choose $\vec{V}_{p}=\vec{V}$ as recovery sequence.

(ii) Let $\vec{V}_{p} \in L^{2}\left(A, \mathbb{R}^{3}\right)$ be functions converging to $\vec{V}$ in $L^{2}\left(A, \mathbb{R}^{3}\right)$, such that

$$
\liminf _{p \rightarrow \infty} F_{p}\left(\vec{V}_{p}\right)<+\infty .
$$

By Lemma 4.7 we have that $\rho_{\Delta}(\operatorname{curl} \vec{V}) \leq 1$ a.e. in $A$, and hence

$$
F(\vec{V})=\int_{A}\left|\vec{V}-\vec{U}_{0}\right|^{2} d x=\lim _{p \rightarrow \infty} \int_{A}\left|\vec{V}_{p}-\vec{U}_{0}\right|^{2} d x \leq \liminf _{p \rightarrow \infty} F_{p}\left(\vec{V}_{p}\right),
$$

concluding the proof.

Notice that the $\Gamma$-convergence result is obtained under the sole assumption that $\vec{U}_{0} \in$ $L^{2}\left(A, \mathbb{R}^{3}\right)$. Actually in our model $\vec{U}_{0}=\vec{H}_{i}^{n}-\vec{H}_{s}\left(t_{i+1}^{n}\right)$ so that $\vec{U}_{0} \in X_{2}+\vec{c}_{0}, \vec{c}_{0} \in \mathbb{R}^{3}$, and $\rho_{\Delta}\left(\operatorname{curl} \vec{U}_{0}\right) \leq 1$. This additional regularity is needed in order to recover the convergence of the minimizers.

Theorem 4.9. Let $\vec{U}_{0} \in \vec{c}_{0}+X_{2}, \vec{c}_{0} \in \mathbb{R}^{3}$, with $\rho_{\Delta}\left(\operatorname{curl} \vec{U}_{0}\right) \leq 1$ a.e. in A. Let $\vec{U}_{p}$ be the unique minimizer of $F_{p}, p>3$, and let $\vec{U}$ be the unique minimizer of $F$. Then $\left(\vec{U}_{p}\right)$ converges to $\vec{U}$ in the weak topology of $W^{1, q}\left(A, \mathbb{R}^{3}\right)$ for every $q>3$.

Proof. Reasoning as in (13), for $3<q<p$ we have

$$
\int_{A}\left|\operatorname{curl} \vec{U}_{p}\right|^{q} d x \leq \frac{1}{c_{1}^{q}}\left(\frac{p}{|A|}\right)^{q / p}|A|\left(\int_{A} \frac{1}{p} \rho_{\Delta}\left(\operatorname{curl} \vec{U}_{p}\right)^{p} d x\right)^{q / p} .
$$


On the other hand, by the minimality of $\vec{U}_{p}$ we get

$$
\int_{A} \frac{1}{p} \rho_{\Delta}\left(\operatorname{curl} \vec{U}_{p}\right)^{p} d x \leq F_{p}\left(\vec{U}_{p}\right) \leq F_{p}\left(\vec{U}_{0}-\vec{c}_{0}\right) \leq\left(1+\lambda\left|\vec{c}_{0}\right|^{2}\right)|A|,
$$

so that

$$
\int_{A}\left|\operatorname{curl} \vec{U}_{p}\right|^{q} d x \leq \frac{1}{c_{1}^{q}}\left[p\left(1+\lambda\left|\vec{c}_{0}\right|^{2}\right)\right]^{q / p}|A| \leq \frac{1}{c_{1}^{q}}|A| \exp \left[q\left(1+\lambda\left|\vec{c}_{0}\right|^{2}\right) / e\right] .
$$

Then the sequence $\left(\vec{U}_{p}\right)$ is bounded in $W^{1, q}\left(A, \mathbb{R}^{3}\right)$. In particular, it is precompact in $L^{2}\left(A, \mathbb{R}^{3}\right)$. Then, by Remark 4.6 we obtain the convergence of $\left(\vec{U}_{p}\right)$ to $\vec{U}$ in $L^{2}\left(A, \mathbb{R}^{3}\right)$. Finally, notice that the previous estimate implies that $\left(\vec{U}_{p}\right)$ converges to $\vec{U}$ in the weak topology of $W^{1, q}$ for every $q>3$.

\section{Cylindrical superconductors}

In what follows we shall consider a long cylindrical type-II superconductor occupying the region $\Omega \times \mathbb{R}$, with a simply connected cross section $\Omega \subset \mathbb{R}^{2}$, placed into a nonstationary uniform axial magnetic field $\vec{H}_{s}(t)=\left(0,0, H_{s}(t)\right)$.

Notice that, due to the parallel geometry, the problem admits a dimensional reduction. Namely, we can assume that there exists a function $u$ depending only on $x=\left(x_{1}, x_{2}\right) \in$ $\Omega$ such that $\vec{H}=\left(0,0, H_{s}(t)+u(x, t)\right)$, so that $\operatorname{curl} \vec{H}=\left(u_{x_{2}}(x, t),-u_{x_{1}}(x, t), 0\right)$ and $\operatorname{div} \vec{H}=0$. In particular the current density $\vec{J}$ is parallel to the cross section plane and its projection on this plane is $\left(J_{1}, J_{2}\right)=(D u)^{\perp}$, where $(D u)^{\perp}$ is the rotation of $-\frac{\pi}{2}$ of $D u$. Hence, under the additional hypothesis that the allowed region $\Delta$ is symmetric with respect to the $z=0$ plane, the constitutive law becomes $D u \in K$, where $K$ is the rotation of the section $z=0$ of the set $\Delta$, and $\vec{E}=\left(E_{1}, E_{2}, 0\right)$ with $\left(E_{1}, E_{2}\right) \in \partial I_{K}(D u)$.

In this case, if $c_{i}^{n}=H_{s}\left(t_{i}^{n}\right)$, and $h_{i}^{n}(x)$ is the magnetic field intensity at time layer $i$, given by $H_{s}\left(t_{i}^{n}\right)+u\left(t_{i}^{n}, x\right)$, the variational formulation is

$$
\min \left\{\int_{\Omega}\left(w-h_{i}^{n}\right)^{2} d x ; w \in c_{i+1}^{n}+W_{0}^{1,1}(\Omega), D w \in K \text { a.e. in } \Omega\right\} .
$$

In the following sections we shall give an explicit representation of the unique minimizer $h_{i+1}^{n}$ and we shall show that the previous quasistatic evolution converges to a function $h(x, t)$ (also explicit), which determines the evolution of the internal magnetic field.

Further to the dimensional reduction and a rescaling, we obtain that $h_{i+1}^{n}-c_{i+1}^{n}$ is the unique minimum point of the functional

$$
J(v)=\int_{\Omega}\left[I_{K}(D v)+(v-\bar{u})^{2}\right] d x, \quad v \in W_{0}^{1,1}(\Omega),
$$

where $\bar{u}=h_{i}^{n}-c_{i+1}^{n}$.

Although in this case we deal with an unbounded superconductor $A=\Omega \times \mathbb{R}$, the above reduction allows us to prove, using the same arguments as in Section 1 (see also [18], Section 2), a $\Gamma$-convergence result, and, consequently, the convergence of the minimizers. Even if the case $N=2$ is the sole meaningful physical situation, the following results hold for every dimension $N \geq 2$.

Theorem 5.1. Let $\Omega \subset \mathbb{R}^{N}$ be a bounded open set, let $K \subset \mathbb{R}^{N}$ be a nonempty, compact, convex set with $0 \in \operatorname{int} K$, and let $\rho_{K}$ be the gauge function of $K$. Let $G_{p}, p \geq 1$, and $G$ 
be the functionals defined respectively by

$$
G_{p}(v)= \begin{cases}\int_{\Omega} \frac{1}{p} \rho_{K}(D v)^{p} d x, & v \in W_{0}^{1, p}(\Omega) \\ +\infty & \text { otherwise in } L^{1}(\Omega)\end{cases}
$$

and

$$
G(v)= \begin{cases}0, & v \in W_{0}^{1, \infty}(\Omega), \rho(D v) \in K \\ +\infty & \text { otherwise in } L^{1}(\Omega) .\end{cases}
$$

Then $\left(G_{p}\right) \Gamma$-converges to $G$ in $L^{r}(\Omega)$ for every $r \geq 1$. As a consequence, for a given $\bar{u} \in L^{2}(\Omega)$ the functionals

$$
J_{p}(v)= \begin{cases}\int_{\Omega}\left[\frac{1}{p} \rho_{K}(D v)^{p} d x+(v-\bar{u})^{2}\right] d x, & v \in W_{0}^{1, p}(\Omega) \\ +\infty & \text { otherwise in } L^{2}(\Omega)\end{cases}
$$

$\Gamma$-converge to the functional

$$
J(v)= \begin{cases}\int_{\Omega}\left[I_{K}(D v)+(v-\bar{u})^{2}\right] d x, & v \in W_{0}^{1,1}(\Omega) \\ +\infty & \text { otherwise in } L^{2}(\Omega),\end{cases}
$$

in $L^{2}(\Omega)$. If in addition $\bar{u} \in c_{0}+W_{0}^{1, \infty}(\Omega), c_{0} \in \mathbb{R}$, with $\rho(D \bar{u}) \leq 1$ a.e. in $\Omega$, then the minimizers $u_{p}$ of $J_{p}$ converge to the unique minimizer $u$ of the functional $J$ in the weak topology of $W_{0}^{1, q}(\Omega)$ for every $q>1$.

\section{The Minkowski Distance function}

Here and hereafter we shall deal with

$\Omega \subset \mathbb{R}^{N}$ nonempty, bounded, open connected set of class $C^{2}$,

and

$$
\begin{aligned}
& K \subset \mathbb{R}^{N} \text { nonempty, compact, convex set, } 0 \in \operatorname{int} K, \\
& \partial K \text { of class } C^{2} \text { with strictly positive principal curvatures . }
\end{aligned}
$$

The polar set of $K$ is defined by

$$
K^{0}=\left\{p \in \mathbb{R}^{N} ;\langle p, x\rangle \leq 1 \forall x \in K\right\} .
$$

We recall that, if $K$ satisfies (19), then $K^{0}$ also satisfies (19), and $K^{00}=\left(K^{0}\right)^{0}=K$ (see [23, Thm. 1.6.1]).

Since $K$ will be kept fixed, from now on we shall use for the gauge functions the notation $\rho=\rho_{K}$ and $\rho^{0}=\rho_{K^{0}}$

It can be readily seen that the gauge function $\rho^{0}$ of the polar set $K^{0}$ coincides with the support function of the set $K$. As a consequence, we have that

$$
\rho\left(D \rho^{0}(\xi)\right)=1 \quad \forall \xi \in \mathbb{R}^{N} \backslash\{0\} .
$$

Let $\operatorname{Lip}_{\rho}(\Omega)$ be the set of functions defined by

$$
\operatorname{Lip}_{\rho}(\Omega):=\{u \in \operatorname{Lip}(\bar{\Omega}): D u \in K \text { a.e. in } \Omega\} .
$$

We recall that $u \in \operatorname{Lip}_{\rho}(\Omega)$ if and only if

$$
u(x)-u(y) \leq \rho^{0}(x-y),
$$

for every $x, y \in \Omega$ joined by a segment contained in $\Omega$. 
The main tools needed in the following sections are the distances from the boundary of $\Omega$ associated to the Minkowski structures induced by the gauge function of $K^{0}$ and $-K^{0}$ respectively.

Definition 6.1. The Minkowski distance from the boundary of $\Omega$ is

$$
d_{\Omega}(x)=\inf _{y \in \partial \Omega} \rho^{0}(x-y), \quad x \in \bar{\Omega} .
$$

Similarly, we define $d_{\Omega}^{-}(x)$ as the distance from the boundary induced by the gauge of $-K^{0}$

Notice that the function $d_{\Omega}^{-}$coincides with $d_{\Omega}$ only if $K^{0}$ is symmetric with respect to the origin. In the remaining part of this section we illustrate some features of the function $d_{\Omega}$. The analogous for $d_{\Omega}^{-}$can be obtained upon observing that $-K^{0}$ is the polar set of $-K$.

Since $\partial \Omega$ is a compact subset of $\mathbb{R}^{N}$ and $\rho^{0}$ is a continuous function, the infimum in the definition of $d_{\Omega}$ is achieved. We shall denote by $\Pi(x)$ the set of projections of $x$ in $\partial \Omega$, that is

$$
\Pi(x)=\left\{y \in \partial \Omega ; d_{\Omega}(x)=\rho^{0}(x-y)\right\}, \quad x \in \bar{\Omega} .
$$

Definition 6.2. We say that $x \in \Omega$ is a regular point of $\Omega$ if $\Pi(x)$ is a singleton. We say that $x \in \Omega$ is a singular point of $\Omega$ if $x$ is not a regular point. We denote by $\Sigma \subseteq \Omega$ the set of all singular points of $\Omega$.

Remark 6.3. It is well known that $d_{\Omega}$ is a Lipschitz function in $\Omega$, and that $d_{\Omega}$ is differentiable at $x \in \Omega$ if and only if $x$ is a regular point. In addition, if $\Pi(x)=\{y\}$ then $D d_{\Omega}(x)=D \rho^{0}(x-y)$, and hence, by (20), $D d_{\Omega} \in K$ almost everywhere in $\Omega$ (see [4, 12, 21]; see also Theorem 6.5(i) below).

Remark 6.4. We recall that $d_{\Omega}$ (resp. $d_{\Omega}^{-}$) is the unique viscosity solution of the HamiltonJacobi equation $\rho(D u)=1$ (resp. $-\rho(D u)=-1$ ) in $\Omega$, with boundary condition $u=0$ on $\partial \Omega$. As a consequence of the maximality property of the viscosity solutions, we have that

$$
-d_{\Omega}^{-} \leq u \leq d_{\Omega}, \quad \forall u \in \operatorname{Lip}_{\rho}(\Omega), u=0 \text { on } \partial \Omega
$$

(see [21]). Moreover it can be easily checked that

$$
\begin{array}{ll}
u \geq-d_{\Omega}^{-}, & \forall u \in \operatorname{Lip}_{\rho}(\Omega), u \geq 0 \text { on } \partial \Omega, \\
u \leq d_{\Omega}, & \forall u \in \operatorname{Lip}_{\rho}(\Omega), u \leq 0 \text { on } \partial \Omega,
\end{array}
$$

(see e.g. 河, Theorem 5.9).

In the following theorem we collect all the results proved in [14 that are relevant for the subsequent analysis.

Theorem 6.5. Assume that $\Omega$ and $K$ satisfy respectively (18) and (19). Then the following hold.

(i) $\bar{\Sigma} \subset \Omega$, and the Lebesgue measure of $\bar{\Sigma}$ is zero.

(ii) Let $x \in \Omega$ and $y \in \Pi(x)$. Then $d_{\Omega}$ is differentiable at every $z$ along the segment jointing $y$ to $x$ (without endpoints), and $D d_{\Omega}(z)=\frac{\nu(y)}{\rho(\nu(y))}$, where $\nu(y)$ is the (Euclidean) inward normal unit vector to $\partial \Omega$ at $y$.

(iii) The function $d_{\Omega}$ is of class $C^{2}$ in $\bar{\Omega} \backslash \bar{\Sigma}$.

Proof. See Remark 4.16, Corollary 6.9, Lemma 4.3 and Theorem 6.10 in [14]. 
At any point $y \in \partial \Omega$ there is a unique inward "normal" direction $p(y)$ with the properties $\Pi(y+t p(y))=\{y\}$ and $d_{\Omega}(y+t p(y))=t$ for $t \geq 0$ small enough (see [14, Remark 4.5]). More precisely, these properties hold true for $p(y)=D \rho(\nu(y))$ and for every $t \in[0, l(y))$, where $l(y)$ is defined by

$$
l(y)=\min \left\{t \geq 0 ; y+t D \rho\left(D d_{\Omega}(x)\right) \in \bar{\Sigma}\right\}
$$

(see [14, Propositions 4.4 and 4.8] and [20, Lemma 2.2]). From Theorem 6.5(ii) and the positive 0-homogeneity of $D \rho$ it is plain that $D \rho(\nu(y))=D \rho\left(D d_{\Omega}(y)\right)$.

From Theorem 6.5(iii), the function $d_{\Omega}$ is of class $C^{2}$ on $\partial \Omega$. Then we can define the function

$$
W(y)=-D^{2} \rho\left(D d_{\Omega}(y)\right) D^{2} d_{\Omega}(y), \quad y \in \partial \Omega .
$$

For any $y \in \partial \Omega$ let $T_{y}$ denote the tangent space to $\partial \Omega$ at $y$. It can be proved that for every $v \in T_{y}$, one has $W(y) v \in T_{y}$. Hence, we can define the map

$$
\bar{W}(y): T_{y} \rightarrow T_{y}, \quad \bar{W}(y) w=W(y) w,
$$

that can be identified with a linear application from $\mathbb{R}^{N-1}$ to $\mathbb{R}^{N-1}$.

Although the matrix $\bar{W}(y)$ is not in general symmetric, its eigenvalues are real numbers, and so its eigenvectors are real (see [14], Remark 5.3). The eigenvalues of $\bar{W}(y)$ have an important geometric interpretation.

Definition 6.6. Let $y \in \partial \Omega$. The anisotropic curvatures of $\partial \Omega$ at $y$, with respect to the Minkowski norm $d_{\Omega}$, are the eigenvalues $\tilde{\kappa}_{1}(y) \leq \cdots \leq \tilde{\kappa}_{n-1}(y)$ of $\bar{W}(y)$.

In what follows we shall denote by $\Pi^{-}, \tilde{\kappa}_{j}^{-}, j=1, \ldots, n-1, \Sigma^{-}$and $l^{-}$, respectively the projection, the anisotropic curvatures and the singular set associated to $d_{\Omega}^{-}$, and the normal distance to the cut locus $\Sigma^{-}$.

\section{A MiNIMUM PROBLEM}

Fixed $\bar{u} \in \operatorname{Lip}_{\rho}(\Omega)$, we want to minimize the functional

$$
J(v)=\int_{\Omega}\left[I_{K}(D v)+(v-\bar{u})^{2}\right] d x, \quad v \in W_{0}^{1,1}(\Omega) .
$$

Let us consider the partition $\Omega=\Omega^{+} \cup \Omega^{-} \cup \Omega^{0}$, with

$$
\Omega^{0}=\left\{-d_{\Omega}^{-} \leq \bar{u} \leq d_{\Omega}\right\}, \Omega^{+}=\left\{\bar{u}>d_{\Omega}\right\}, \Omega^{-}=\left\{\bar{u}<-d_{\Omega}^{-}\right\},
$$

and define the function

$$
u(x)= \begin{cases}d_{\Omega}(x) & x \in \Omega^{+} \\ -d_{\Omega}^{-}(x) & x \in \Omega^{-} \\ \bar{u}(x) & x \in \Omega^{0}\end{cases}
$$

The aim of this section is to show that $u$ is the unique minimizer of $J$ in $W_{0}^{1,1}(\Omega)$.

Remark 7.1. If $x \in \bar{\Omega}^{+} \cap \bar{\Omega}^{-}$, then $\bar{u}(x)=d_{\Omega}(x)=-d_{\Omega}^{-}(x)$ and hence $x \in \partial \Omega$ and $\bar{u}(x)=0$. As a consequence, we have $u(x)=\min \left(\left(\max \left(\bar{u}(x),-d_{\Omega}^{-}(x)\right), d_{\Omega}(x)\right)\right)$. This implies that $u \in \operatorname{Lip}_{\rho}(\Omega), u=0$ on $\partial \Omega, \rho(D u)=1$ a.e. in $\Omega^{+} \cup \Omega^{-}$, and $u$ is a viscosity solution of $\rho(D u)=1$ in $\Omega^{+}$and of $-\rho(D u)=-1$ in $\Omega^{-}$. 
Let us define the following two subsets of $\partial \Omega$ :

$$
\Gamma^{+}=\{y \in \partial \Omega: \bar{u}(y)>0\}, \quad \Gamma^{-}=\{y \in \partial \Omega: \bar{u}(y)<0\},
$$

and the functions

$$
\begin{aligned}
\lambda(y) & =\sup \{s \in[0, l(y)): \bar{u}(y+t D \rho(\nu(y)))>t \forall t \in[0, s)\}, \\
\lambda^{-}(y) & =\sup \left\{s \in\left[0, l^{-}(y)\right): \bar{u}\left(y+t D \rho_{-}(\nu(y))\right)<-t \forall t \in[0, s)\right\} .
\end{aligned}
$$

Recall that $l(y)$ and $l^{-}(y)$ are the normal distance to the cut locus, corresponding to $d_{\Omega}$ and $d_{\Omega}^{-}$respectively, that is the length of the "normal" ray starting from $y$ and ending on $\bar{\Sigma}($ see $(24))$.

Remark 7.2. It can be easily checked that $\lambda$ and $\lambda^{-}$are lower semicontinuous function in $\partial \Omega$. In general we cannot expect the continuity of these functions, as it is shown by the following example. Let $\Omega=B_{1}(0) \subseteq \mathbb{R}^{2}, K=\bar{B}_{1}(0) \subseteq \mathbb{R}^{2}$, and define $\bar{u}(r \cos \theta, r \sin \theta)=$ $1-r \cos \theta$ for $0 \leq r<1, \theta \in[0,2 \pi)$. We have $|D \bar{u}(r \cos \theta, r \sin \theta)|^{2}=\cos ^{2} \theta+\sin ^{2} \theta=1$ $(0<r<1)$, so that $\bar{u} \in \operatorname{Lip}_{\rho}(\Omega)$. Moreover, if $y=(\cos \theta, \sin \theta) \in \partial \Omega, \theta \in[0,2 \pi), \lambda$ is the lower semicontinuous function given by

$$
\lambda(y)= \begin{cases}0, & \theta=0, \\ 1, & \theta \in(0,2 \pi) .\end{cases}
$$

The following simple lemma provides a characterization of $\Omega^{+}$and $\Omega^{-}$in terms of the rays starting from points of $\Gamma^{+}$and $\Gamma^{-}$respectively.

Lemma 7.3. Let $y \in \Gamma^{+}$be such that $\lambda(y)<l(y)$. Then $y+t D \rho(\nu(y)) \in \Omega^{0}$ for every $t \in$ $[\lambda(y), l(y)]$. Analogously, let $y \in \Gamma^{-}$be such that $\lambda^{-}(y)<l^{-}(y)$. Then $y+t D \rho_{-}(\nu(y)) \in \Omega^{0}$ for every $t \in\left[\lambda^{-}(y), l^{-}(y)\right]$. As a consequence, we have

$$
\begin{aligned}
& \Omega^{+} \backslash \bar{\Sigma}^{+}=\left\{y+t D \rho(\nu(y)): y \in \Gamma^{+}, t \in(0, \lambda(y))\right\}, \\
& \Omega^{-} \backslash \bar{\Sigma}^{-}=\left\{y+t D \rho_{-}(\nu(y)): y \in \Gamma^{-}, t \in\left(0, \lambda^{-}(y)\right)\right\} .
\end{aligned}
$$

Proof. Let $y \in \Gamma^{+}$and assume that $\lambda(y)<l(y)$. From the very definition of $\lambda(y)$ and the continuity of $\bar{u}$, we have that $\bar{u}(y+\lambda(y) D \rho(\nu(y)))=\lambda(y)$. For every $t \in[\lambda(y), l(y)]$ we have that

$$
d_{\Omega}(y+t D \rho(\nu(y)))=t=\bar{u}(y+\lambda(y) D \rho(\nu(y)))+t-\lambda(y) \geq \bar{u}(y+t D \rho(\nu(y))),
$$

where the last inequality follows from the assumption $\bar{u} \in \operatorname{Lip}_{\rho}(\Omega)$ (see (21)), and from the fact that $\rho^{0}((t-\lambda) D \rho(\nu(y)))=t-\lambda($ see $(20))$. The inequality $\bar{u}(y+t D \rho(\nu(y))) \geq$ $-d_{\Omega}^{-}(y+t D \rho(\nu(y)))$ follows easily from the fact that $\bar{u}(y)>0$ and $\bar{u} \in \operatorname{Lip}_{\rho}(\Omega)$ (see Remark 6.4). The case $y \in \Gamma^{-}$can be handled in a similar way.

The following change of variable formula can be proved as in [14, Theorem 7.1].

Theorem 7.4. Let $\Phi, \Phi^{-}: \partial \Omega \times \mathbb{R} \rightarrow \mathbb{R}^{N}$ be the maps defined respectively by

$$
\Phi(y, t)=y+t D \rho(\nu(y)), \quad \Phi^{-}(y, t)=y+t D \rho_{-}(\nu(y)), \quad(y, t) \in \partial \Omega \times \mathbb{R} .
$$

Then

$$
\begin{aligned}
\int_{\Omega^{+}} h(x) d x & =\int_{\Gamma^{+}} \rho(\nu(y))\left[\int_{0}^{\lambda(y)} h(\Phi(y, t)) \prod_{i=1}^{n-1}\left(1-t \tilde{\kappa}_{i}(y)\right) d t\right] d \mathcal{H}^{n-1}(y) \\
\int_{\Omega^{-}} h(x) d x & =\int_{\Gamma^{-}} \rho_{-}(\nu(y))\left[\int_{0}^{\lambda^{-}(y)} h\left(\Phi^{-}(y, t)\right) \prod_{i=1}^{n-1}\left(1-t \tilde{\kappa}_{i}^{-}(y)\right) d t\right] d \mathcal{H}^{n-1}(y)
\end{aligned}
$$


for every $h \in L^{1}(\Omega)$.

In what follows we will set

$$
M_{x}(t)=\prod_{i=1}^{n-1} \frac{1-t \tilde{\kappa}_{i}(x)}{1-d_{\Omega}(x) \tilde{\kappa}_{i}(x)}, \quad M_{x}^{-}(t)=\prod_{i=1}^{n-1} \frac{1-t \tilde{\kappa}_{i}^{-}(x)}{1-d_{\Omega}^{-}(x) \tilde{\kappa}_{i}^{-}(x)} .
$$

From Lemma 7.3 in [14] we deduce that there exists a positive constant $M_{0}$ such that

$$
0 \leq M_{x}(t) \leq M_{0} \quad \forall x \in \Omega \backslash \bar{\Sigma}, d_{\Omega}(x) \leq t<l(y),
$$

where $\Pi(x)=\{y\}$. An analogous estimate holds for $M_{x}^{-}(t)$.

Using Theorem 7.4 and (31), we are able to show that the function $u$ defined in (28), coupled with an explicit function $v$, solves a system of PDEs of Monge-Kantorowich type. A posteriori this system will be understood as a first order necessary condition fulfilled by the minimizer of $J$.

Lemma 7.5. Let $u \in \operatorname{Lip}_{\rho}(\Omega)$ be the function defined in (28), and let $v: \Omega \rightarrow \mathbb{R}$ be the function defined by

$$
v(x)= \begin{cases}\int_{d_{\Omega}(x)}^{\lambda(y)}\left[\bar{u}\left(y+t D \rho\left(D d_{\Omega}(x)\right)\right)-t\right] M_{x}(t) d t, & x \in \Omega^{+} \backslash \bar{\Sigma}^{+}, \Pi(x)=\{y\} \\ -\int_{d_{\Omega}^{-}(x)}^{\lambda^{-}(y)}\left[\bar{u}\left(y+t D \rho_{-}\left(D d_{\Omega}(x)\right)\right)+t\right] M_{x}^{-}(t) d t, & x \in \Omega^{-} \backslash \bar{\Sigma}^{-}, \Pi^{-}(x)=\{y\} \\ 0 & \text { otherwise. }\end{cases}
$$

Then $v \in C_{b}(\Omega), v \geq 0$, and the pair $(u, v)$ solves the system of PDEs

$$
\begin{cases}-\operatorname{div}(v D \rho(D u))=\bar{u}-u & \text { in } \Omega, \quad \text { (distributional) } \\ \rho(D u) \leq 1 & \text { a.e. in } \Omega, \\ \rho(D u)=1 & \text { a.e. in }\{v>0\}, \\ u=0 & \text { in } \partial \Omega .\end{cases}
$$

Proof. Let us extend the functions $\lambda$ and $\lambda^{-}$to $\Omega^{+} \backslash \Sigma^{+}$and $\Omega^{-} \backslash \Sigma^{-}$respectively by setting $\lambda(x):=\lambda(y)$ when $\Pi(x)=\{y\}$ and $\lambda^{-}(x):=\lambda^{-}(y)$ when $\Pi^{-}(x)=\{y\}$. Since $\lambda(x) \geq d_{\Omega}(x)$ for every $x \in \Omega^{+} \backslash \Sigma^{+}$and $\lambda^{-}(x) \geq d_{\Omega}^{-}(x)$ for every $x \in \Omega^{-} \backslash \Sigma^{-}$, it is plain that $v \geq 0$.

Let us prove that $v$ is a continuous function. It is convenient to rewrite $v$ in $\Omega^{+} \backslash \bar{\Sigma}^{+}$ in the following way:

$$
v(x)=\int_{d_{\Omega}(x)}^{l(y)}\left[t \vee \bar{u}\left(y+t D \rho\left(D d_{\Omega}(x)\right)\right)-t\right] M_{x}(t) d t, \quad x \in \Omega^{+} \backslash \bar{\Sigma}^{+}, \Pi(x)=\{y\} .
$$

Since the maps $d_{\Omega}, D d_{\Omega}, \bar{u}, l$, and $x \mapsto \Pi(x)$ are continuous in $\Omega^{+} \backslash \bar{\Sigma}^{+}$, it follows that also $v$ is continuous in $\Omega^{+} \backslash \bar{\Sigma}^{+}$.

If $x_{0} \in \Omega^{+} \cap \bar{\Sigma}^{+}$, then $\lambda\left(x_{0}\right)=l\left(x_{0}\right)=d_{\Omega}\left(x_{0}\right)$, and the map $\lambda$ is continuous at $x_{0}$. Namely, since $x_{0} \in \bar{\Sigma}^{+}$, we have that $l\left(x_{0}\right)=d_{\Omega}\left(x_{0}\right)$. Moreover, since $x_{0} \in \Omega^{+}$, we have that $\bar{u}\left(x_{0}\right)>d_{\Omega}\left(x_{0}\right)$, so that $\bar{u}>d_{\Omega}$ on every segment $\left[y, x_{0}\right] \subset \bar{\Omega}$ with $y \in \partial \Omega$. Thus $\lambda\left(x_{0}\right) \geq d_{\Omega}\left(x_{0}\right)$. Finally, from the lower semicontinuity of $\lambda$ and the fact that $\lambda(x) \leq l(x)$ for every $x \in \Omega$, we conclude that

$$
d_{\Omega}\left(x_{0}\right) \leq \lambda\left(x_{0}\right) \leq \liminf _{x \rightarrow x_{0}} \lambda(x) \leq \limsup _{x \rightarrow x_{0}} \lambda(x) \leq \lim _{x \rightarrow x_{0}} l(x)=l\left(x_{0}\right)=d_{\Omega}\left(x_{0}\right),
$$


so that $\lambda\left(x_{0}\right)=d_{\Omega}\left(x_{0}\right)=\lim _{x \rightarrow x_{0}} \lambda(x)$.

From the very definition of $v$ and (32) we have that

$$
0 \leq v(x) \leq C\left[\lambda(x)-d_{\Omega}(x)\right] \quad \forall x \in \Omega^{+} \backslash \bar{\Sigma}^{+} .
$$

We remark that the same estimate trivially holds also on $\Omega^{+} \cap \bar{\Sigma}^{+}$since $v$ vanishes. If $x_{0} \in \Omega^{+} \cap \bar{\Sigma}^{+}$, we have that

$$
\limsup _{x \rightarrow x_{0}} v(x) \leq \limsup _{x \rightarrow x_{0}} C\left[\lambda(x)-d_{\Omega}(x)\right]=0,
$$

hence $\lim _{x \rightarrow x_{0}} v(x)=0=v\left(x_{0}\right)$.

Up to now we have proved that $v$ is continuous in $\Omega^{+}$. A similar argument shows that $v$ is continuous in $\Omega^{-}$. Since $v$ vanishes on $\Omega^{0}$ and $\partial \Omega^{+} \cap \partial \Omega^{-} \cap \Omega=\emptyset$ (see Remark 7.1), it remains to prove that $v$ is continuous on $\Omega \cap \partial \Omega^{+}$and $\Omega \cap \partial \Omega^{-}$.

Let $x_{0} \in \Omega \cap \partial \Omega^{+}$. Since $x_{0} \in \Omega^{0}$, we have that $v\left(x_{0}\right)=0$. Moreover, by definition of $\Omega^{+}, \bar{u}\left(x_{0}\right)=d_{\Omega}\left(x_{0}\right)$. Since $\bar{u} \in \operatorname{Lip}_{\rho}(\Omega)$, by (21) and (2), given $\epsilon>0$ we have that

$$
\bar{u}(x) \leq d_{\Omega}(x)+2 c_{2} \epsilon \quad \forall x \in \Omega \cap B_{\epsilon}\left(x_{0}\right) .
$$

Moreover, if $x \in\left(\Omega^{+} \cap B_{\epsilon}\left(x_{0}\right)\right) \backslash \bar{\Sigma}^{+}$, we have that $\bar{u} \leq d_{\Omega}+2 c_{2} \epsilon$ along the segment joining $x$ to its cut point. As a consequence, the integrand in (35) is bounded from above by $2 c_{2} \in M_{0}$, so that

$$
0 \leq v(x) \leq C \epsilon \quad \forall x \in\left(\Omega^{+} \cap B_{\epsilon}\left(x_{0}\right)\right) \backslash \bar{\Sigma}^{+} .
$$

Since $v=0$ in $\Omega^{0} \cup \bar{\Sigma}^{+}$, this inequality clearly implies that

$$
\lim _{x \rightarrow x_{0}} v(x)=0=v\left(x_{0}\right),
$$

i.e. $v$ is continuous at $x_{0}$. The continuity in $\Omega \cap \partial \Omega^{-}$can be proved in a similar way.

Let us prove that the pair $(u, v)$ is a solution to (34). Upon observing that $\{v>0\}=$ $\Omega^{+} \cup \Omega^{-}$, by Remark 7.1 we have only to prove that $(u, v)$ solves

$$
\int_{\Omega} v\langle D \rho(D u), D \varphi\rangle d x=\int_{\Omega}(\bar{u}-u) \varphi d x
$$

for all $\varphi \in C_{0}^{\infty}(\Omega)$. We have that

$$
\int_{\Omega}(\bar{u}-u) \varphi d x=\int_{\Omega^{+}}(\bar{u}-u) \varphi d x+\int_{\Omega^{-}}(\bar{u}-u) \varphi d x .
$$

By using the change of variables stated in Theorem 7.4, we get

$$
\int_{\Omega^{-}}(\bar{u}-u) \varphi=\int_{\Gamma^{-}} \rho_{-}(\nu)\left[\int_{0}^{\lambda^{-}}\left(\bar{u}\left(\Phi^{-}\right)-u\left(\Phi^{-}\right)\right) \varphi\left(\Phi^{-}\right) \prod_{i=1}^{n-1}\left(1-t \tilde{\kappa}_{i}^{-}\right) d t\right] d \mathcal{H}^{n-1} .
$$

An integration by parts leads to

$$
\begin{aligned}
& \int_{0}^{\lambda^{-}}\left(\bar{u}\left(\Phi^{-}\right)-u\left(\Phi^{-}\right)\right) \varphi\left(\Phi^{-}\right) \prod_{i=1}^{n-1}\left(1-t \tilde{\kappa}_{i}^{-}\right) d t \\
& =\int_{0}^{\lambda^{-}}\left\langle D \varphi\left(\Phi^{-}\right), D \rho_{-}(\nu)\right\rangle\left[\int_{t}^{\lambda^{-}}\left(\bar{u}\left(\Phi^{-}\right)-u\left(\Phi^{-}\right)\right) \prod_{i=1}^{n-1}\left(1-s \tilde{\kappa}_{i}^{-}\right) d s\right] d t .
\end{aligned}
$$

Since $u\left(\Phi^{-}(y, s)\right)=-s$ for every $s \in\left[0, \lambda^{-}(y)\right]$, we have that

$$
-v\left(\Phi^{-}(y, t)\right)=\int_{t}^{\lambda^{-}(y)}\left(\bar{u}\left(\Phi^{-}(y, s)\right)-u\left(\Phi^{-}(y, s)\right)\right) \prod_{i=1}^{n-1} \frac{1-s \tilde{\kappa}_{i}^{-}(y)}{1-t \tilde{\kappa}_{i}^{-}(y)} d s
$$


for every $y \in \Gamma^{-}$and $t \in\left[0, \lambda^{-}(y)\right)$. Then we obtain

$$
\int_{\Omega^{-}}(\bar{u}-u) \varphi d x=-\int_{\Gamma^{-}} \rho_{-}(\nu)\left[\int_{0}^{\lambda^{-}}\left\langle D \varphi\left(\Phi^{-}\right), D \rho_{-}(\nu)\right\rangle v(\Phi) \prod_{i=1}^{n-1}\left(1-t \tilde{\kappa}_{i}^{-}\right) d t\right] d \mathcal{H}^{n-1} .
$$

Finally, again from Theorem 7.4 and the fact that

$$
D \rho_{-}(\nu(y))=-D \rho(-\nu(y))=-D \rho\left(-D d_{\Omega}^{-}(y)\right)=-D \rho(D u(y)),
$$

we get

$$
\int_{\Omega^{-}}(\bar{u}-u) \varphi d x=\int_{\Omega^{-}} v\langle D \rho(D u), D \varphi\rangle d x,
$$

for every $\varphi \in C_{c}^{\infty}(\Omega)$. In the same way, using the change of variables in $\Omega^{+}$, we get

$$
\int_{\Omega^{+}}(\bar{u}-u) \varphi=\int_{\Omega^{+}} v\langle D \rho(D u), D \varphi\rangle,
$$

for every $\varphi \in C_{c}^{\infty}(\Omega)$, concluding the proof.

Now the absolute minimality of $u$ follows easily.

Theorem 7.6. The function $u$ defined in (28) is the unique minimizer of $J$ in $W_{0}^{1,1}(\Omega)$.

Proof. Let $v$ be the function defined in (33). Since $v \geq 0$, for almost every $x \in \Omega^{+} \cup \Omega^{-}$ the vector $2 v(x) D \rho(D u(x))$ belongs to the subdifferential of $I_{K}$ at $D u(x)$ (see (ब)). On the other hand, $v=0$ in $\Omega^{0}$. Then we have

$$
I_{K}(D w(x))-I_{K}(D u(x)) \geq 2 v(x)\langle D \rho(D u(x)), D w(x)-D u(x)\rangle \text { a.e. in } \Omega,
$$

for every $w \in W_{0}^{1,1}(\Omega)$. Hence, using Lemma 7.5 we obtain that for every $w \in W_{0}^{1,1}(\Omega)$

$$
\begin{aligned}
J(w)-J(u) & \geq \int_{\Omega} 2 v\langle D \rho(D u), D w-D u\rangle+\int_{\Omega}(w-\bar{u})^{2}+\int_{\Omega}(u-\bar{u})^{2} \\
= & \int_{\Omega}\left[2(w-u)(\bar{u}-u)+(w-\bar{u})^{2}-(u-\bar{u})^{2}\right] \\
= & \int_{\Omega}(u-w)^{2},
\end{aligned}
$$

which implies that $u$ is the unique minimizer of $J$ in $W_{0}^{1,1}(\Omega)$.

\section{The QUASISTATIC EVOLUtion}

Let $T>0$ be fixed. For every $n \in \mathbb{N}^{+}$, we take a partition $P^{n}=\left\{t_{i}^{n}\right\}_{i=0}^{k(n)}$ of the interval $[0, T]$, and we set $\delta t_{i}^{n}=t_{i}^{n}-t_{i-1}^{n}$.

The starting configuration of the superconductor at time $t=0$ is given by $h(x, 0)=$ $h_{0}(x) \in \operatorname{Lip}_{\rho}(\Omega)$, with $h_{0}=H_{s}(0)$ on $\partial \Omega$. Using the variational formulation (17) and Theorem 7.6, we have that the internal magnetic field $h_{i+1}^{n}(x)=h\left(x, t_{i+1}^{n}\right)$ is given by

$$
h_{i+1}^{n}(x)=\min \left[\max \left[h_{i}^{n}(x), c_{i+1}^{n}-d_{\Omega}^{-}(x)\right], c_{i+1}^{n}+d_{\Omega}(x)\right] .
$$

Since the model is valid only when the external field varies slowly in time, it is not restrictive to assume that $H_{s}$ is a continuous function obtained by the concatenation of a finite number of monotone functions of class $C^{1}$. Hence from now on we shall consider only the case

$$
H_{s} \text { is a monotone function of class } C^{1}([0, T]) .
$$


In this case formula (37) simplifies. Namely, if we assume that $H_{s}$ is a nondecreasing function we have that $c_{i}^{n} \leq c_{i+1}^{n}$. In particular, at the first time step we have

$$
h_{1}^{n}(x)=\min \left[\max \left[h_{0}(x), c_{1}^{n}-d_{\Omega}^{-}(x)\right], c_{1}^{n}+d_{\Omega}(x)\right],
$$

and $h_{0}(x)-c_{1}^{n} \in \operatorname{Lip}_{\rho}(\Omega)$ with $h_{0}(x)-c_{1}^{n}=c_{0}^{n}-c_{1}^{n} \leq 0$ on $\partial \Omega$. Hence $h_{0}(x)-c_{1}^{n} \leq d_{\Omega}(x)$ in $\Omega$ (see Remark 6.4), which implies

$$
h_{1}^{n}(x)=\max \left[h_{0}(x), c_{1}^{n}-d_{\Omega}^{-}(x)\right] .
$$

At the second time step we get

$$
h_{2}^{n}(x)=\min \left[\max \left[h_{1}^{n}(x), c_{2}^{n}-d_{\Omega}^{-}(x)\right], c_{2}^{n}+d_{\Omega}(x)\right] .
$$

Since $h_{1}^{n}(x)-c_{2}^{n}=\max \left[h_{0}(x)-c_{2}^{n},-d_{\Omega}^{-}(x)+c_{1}^{n}-c_{2}^{n}\right]$, with $c_{1}^{n}-c_{2}^{n} \leq 0$, we have that

$$
h_{2}^{n}(x)=\max \left[h_{0}(x), c_{2}^{n}-d_{\Omega}^{-}(x)\right] .
$$

In general we get

$$
h_{i}^{n}(x)=\max \left[h_{0}(x), c_{i}^{n}-d_{\Omega}^{-}(x)\right] . \quad \text { (nondecreasing external field) }
$$

With a similar argument, we obtain that if $H_{s}$ is nonincreasing in time, the $i$-th time step leads to

$$
h_{i}^{n}(x)=\min \left[h_{0}(x), c_{i}^{n}+d_{\Omega}(x)\right] . \quad \text { (nonincreasing external field) }
$$

Now we easily obtain the evolution of the internal magnetic field, taking the limit as $n$ goes to $+\infty$ of the quasistatic approximation

$$
h^{n}(x, t)=h_{i}^{n}(x), t \in\left[t_{i}^{n}, t_{i+1}^{n}\right) .
$$

Theorem 8.1. If $H_{s}$ is a nondecreasing function in $[0, T]$, then the sequence $\left(h^{n}(x, t)\right)$ converges uniformly in $\bar{\Omega} \times[0, T]$ to

$$
h(x, t)=\max \left[h_{0}(x),-d_{\Omega}^{-}(x)+H_{s}(t)\right] .
$$

If $H_{s}$ is a nonincreasing function in $[0, T]$, then the sequence $\left(h^{n}(x, t)\right)$ converges uniformly in $\bar{\Omega} \times[0, T]$ to

$$
h(x, t)=\min \left[h_{0}(x), d_{\Omega}(x)+H_{s}(t)\right] .
$$

Proof. Assume that $H_{s}$ is nondecreasing (the other case being similar). Given $t \in[0, T]$, and $n \in \mathbb{N}$, we have that $t \in\left[t_{i}^{n}, t_{i+1}^{n}\right)$, and

$$
0 \leq h(x, t)-h^{n}(x, t) \leq H_{s}(t)-H_{s}\left(t_{i}^{n}\right) \leq H_{s}\left(t_{i+1}^{n}\right)-H_{s}\left(t_{i}^{n}\right) \leq \delta t^{n} \max _{t \in[0, T]} H_{s}^{\prime}(t),
$$

where $\delta t^{n}$ is the size of the partition $P^{n}$. Hence the uniform convergence in $\bar{\Omega} \times[0, T]$ follows.

Remark 8.2. Theorem 8.1 shows that the evolution obtained by the direct optimization method at discrete times proposed by Badía and López in [1] coincides with the one obtained in [5, 6] by Barrett and Prigozhin as a solution of an evolutionary variational inequality.

For what concerns the electric field induced in the superconductor, we already know that $\vec{E}=\left(E_{1}, E_{2}, 0\right)$ with $\left(E_{1}, E_{2}\right) \in \partial I_{K}(D h)$. This means that $\vec{E}=0$ whenever $D h \in \operatorname{int} K$, and there exists $w(x, t) \geq 0$ such that $\left(E_{1}, E_{2}\right)=w(x, t) D \rho(D h(x, t))$ for almost every $x \in \Omega$ and for every $t \in[0, T]$. Our approach allows us to compute explicitly the quasistatic evolution of the dissipated power $w(x, t)$ as the dual function appearing in the necessary conditions stated in Lemma 7.5 . 
Namely, from Lemma 7.5, for every $n \in \mathbb{N}$ and for every $i=1, \ldots, k(n)$, there exists $v_{i}^{n} \in C_{b}(\Omega)$ such that

$$
-\operatorname{div}\left(\frac{v_{i}^{n}}{\delta t_{i}^{n}} D \rho\left(D h_{i}^{n}\right)\right)=\frac{h_{i-1}^{n}-h_{i}^{n}}{\delta t_{i}^{n}},
$$

in the sense of distributions in $\Omega$. For our convenience, we also set $v_{0}^{n}=0$. The function $v_{i}^{n}$ is unique, as stated in the following result.

Proposition 8.3. Let $v \in C_{b}(\Omega)$ be a function such that

$$
\begin{cases}-\operatorname{div}\left(v D \rho\left(D h_{i}^{n}\right)\right)=h_{i-1}^{n}-h_{i}^{n}, & \text { in } \Omega(\text { distributional), } \\ \rho\left(D h_{i}^{n}\right)=1 & \text { a.e. in }\{v>0\} .\end{cases}
$$

If $c_{i}^{n} \geq c_{i-1}^{n}$, then $v=v_{i}^{n}$, where

$$
v_{i}^{n}(x)= \begin{cases}\int_{d_{\Omega}^{-}(x)}^{l^{-}(y)}\left(h_{i}^{n}-h_{i-1}^{n}\right)\left(y+s D \rho_{-}(\nu(y))\right) M_{x}^{-}(s) d s, & x \in \Omega \backslash \bar{\Sigma}^{-}, \Pi^{-}(x)=\{y\}, \\ 0 & x \in \bar{\Sigma}^{-}\end{cases}
$$

whereas, if $c_{i}^{n} \leq c_{i-1}^{n}$, then $v=v_{i}^{n}$, where

$$
v_{i}^{n}(x)= \begin{cases}\int_{d_{\Omega}(x)}^{l(y)}\left(h_{i-1}^{n}-h_{i}^{n}\right)(y+s D \rho(\nu(y))) M_{x}(s) d s, & x \in \Omega \backslash \bar{\Sigma}, \Pi(x)=\{y\}, \\ 0 & x \in \bar{\Sigma} .\end{cases}
$$

Proof. Assume that $c_{i-1}^{n}-c_{i}^{n} \geq 0$ (the other case being similar), and let $v \in C_{b}(\Omega)$ be a solution of (42). By construction we have that the function $f:=h_{i-1}^{n}-h_{i}^{n}$ is bounded, continuous and nonnegative in $\Omega$, and $h_{i}^{n}=d_{\Omega}$ in $\operatorname{supp}(f)$. Using the same arguments of Lemma 6.3 in [15], we can prove that the pair $\left(d_{\Omega}, v\right)$ satisfies

$$
-\operatorname{div}\left(v D \rho\left(D d_{\Omega}\right)\right)=f \text {, }
$$

in the sense of distributions in $\Omega$. Now we can apply Propositions 6.5 and 6.7 in 15 in order to conclude that $v=v_{i}^{n}$.

Formula (41) can be understood as a discretized version of Faraday's law, so that the functions $w_{i}^{n}=\frac{v_{i}^{n}}{\delta t_{i}^{n}}$ are the steps of the quasistatic evolution of the dissipated power. If we set

$$
w^{n}(x, t)=w_{i}^{n}(x), \quad g^{n}(x, t)=\frac{h_{i-1}^{n}(x)-h_{i}^{n}(x)}{\delta t_{i}^{n}}, \quad t \in\left[t_{i}^{n}, t_{i+1}^{n}\right)
$$

and $w^{n}(t) \equiv w^{n}(\cdot, t), h^{n}(t) \equiv h^{n}(\cdot, t), g^{n}(t) \equiv g^{n}(\cdot, t)$, formula (41) can be rewritten as

$$
-\operatorname{div}\left(w^{n}(t) D \rho\left(D h^{n}(t)\right)\right)=g^{n}(t) .
$$

Theorem 8.4. If $H_{s}$ is a nondecreasing function in $[0, T]$, then the sequence $\left(w^{n}(x, t)\right)$ converges pointwise in $\bar{\Omega} \times[0, T]$ to

$$
w(x, t)=\frac{\partial H_{s}}{\partial t}(t) \int_{d_{\Omega}^{-}(x)}^{l^{-}(y)} \chi_{\left\{h_{0}\left(y+s D \rho_{-}(\nu(y))\right) \leq H_{s}(t)-s\right\}} M_{x}^{-}(s) d s .
$$

If $H_{s}$ is a nonincreasing function in $[0, T]$, then the sequence $\left(w^{n}(x, t)\right)$ converges pointwise in $\bar{\Omega} \times[0, T]$ to

$$
w(x, t)=-\frac{\partial H_{s}}{\partial t}(t) \int_{d_{\Omega}(x)}^{l(y)} \chi_{\left\{h_{0}(y+s D \rho(\nu(y))) \geq H_{s}(t)+s\right\}} M_{x}(s) d s .
$$


Moreover, in both cases, the sequence $\left(w^{n}\right)$ converges to $w$ in the strong topology of $L^{p}(\Omega)$, $p \geq 1$, uniformly in $[0, T]$.

Proof. We compute the limit of the sequence $\left(w^{n}\right)$ only in the case of nondecreasing external field, the other case being similar.

We can assume, without loss of generality, that for every $n \in \mathbb{N}$ the partition $P^{n+1}$ is a refinement of the partition $P^{n}$. Hence, for every $t \in[0, T]$ and for every $n \in \mathbb{N}$ there exists $i=i(n) \in \mathbb{N}$ such that $t \in\left[t_{i(n)}^{n}, t_{i(n)+1}^{n}\right)$. Moreover, since $H_{s} \in C^{1}([0, T])$, we obtain

$$
-\frac{\partial H_{s}}{\partial t}(t)=\lim _{n \rightarrow \infty} \frac{H_{s}\left(t_{i-1}^{n}\right)-H_{s}\left(t_{i}^{n}\right)}{\delta t_{i}^{n}} \quad \text { uniformly in }[0, T] .
$$

By Proposition 8.3 we get

$$
w^{n}(x, t)=\int_{d_{\Omega}^{-}(x)}^{l^{-}(y)} g^{n}\left(y+s D \rho_{-}(\nu(y)), t\right) M_{x}^{-}(s) d s,
$$

where $g^{n}$ is the function defined in (43). Moreover we have

$$
\begin{aligned}
h_{i-1}^{n}-h_{i}^{n} & =\left(H_{s}\left(t_{i-1}^{n}\right)-H_{s}\left(t_{i}^{n}\right)\right) \chi_{\left\{h_{0} \leq H_{s}\left(t_{i-1}^{n}\right)-d_{\Omega}^{-}\right\}} \\
& +\left(h_{0}-H_{s}\left(t_{i}^{n}\right)+d_{\Omega}^{-}\right) \chi_{\left\{H_{s}\left(t_{i-1}^{n}\right)-d_{\Omega}^{-}<h_{0}<H_{s}\left(t_{i}^{n}\right)-d_{\Omega}^{-}\right\}},
\end{aligned}
$$

and

$$
\begin{aligned}
0 & \geq\left(h_{0}-H_{s}\left(t_{i}^{n}\right)+d_{\Omega}^{-}\right) \chi_{\left\{H_{s}\left(t_{i-1}^{n}\right)-d_{\Omega}^{-}<h_{0}<H_{s}\left(t_{i}^{n}\right)-d_{\Omega}^{-}\right\}} \\
& \geq\left(H_{s}\left(t_{i-1}^{n}\right)-H_{s}\left(t_{i}^{n}\right)\right) \chi_{\left\{H_{s}\left(t_{i-1}^{n}\right)-d_{\Omega}^{-}<h_{0}<H_{s}\left(t_{i}^{n}\right)-d_{\Omega}^{-}\right\}} .
\end{aligned}
$$

Collecting the previous information, we get

$$
\lim _{n \rightarrow+\infty} g^{n}(t)=g(t):=-\frac{\partial H_{s}}{\partial t}(t) \chi_{\left\{h_{0} \leq H_{s}(t)-d_{\Omega}^{-}\right\}},
$$

and $\lim _{n \rightarrow+\infty}\left\|g^{n}(t)-g(t)\right\|_{L^{p}(\Omega)}=0$ uniformly in $[0, T]$, for every $p \geq 1$. In addition, from (45) and (46), for every regular point $x$ of $d_{\Omega}^{-}$and setting $\{y\}=\Pi(x)$, we get

$$
\lim _{n \rightarrow+\infty} g^{n}\left(y+s D \rho_{-}(\nu(y)), t\right)=g\left(y+s D \rho_{-}(\nu(y)), t\right) \quad \text { a.e. } s \in\left[d_{\Omega}^{-}(x), l^{-}(y)\right] .
$$

Finally, by (48), (32) and the Dominated Convergence Theorem, we conclude that

$$
\begin{aligned}
& \lim _{n \rightarrow+\infty} w^{n}(x, t)= \\
& -\frac{\partial H_{s}}{\partial t}(t) \int_{d_{\Omega}^{-}(x)}^{l(y)} \chi_{\left\{h_{0}\left(y+s D \rho_{-}(\nu(y))\right) \leq H_{s}(t)-s\right\}} M_{x}^{-}(t) d s:=w(x, t) .
\end{aligned}
$$

The last part of the theorem follows from the fact that

$$
\left\|w^{n}(t)-w(t)\right\|_{L^{p}(\Omega)} \leq C_{0}\left\|g^{n}(t)-g(t)\right\|_{L^{p}(\Omega)}
$$

for some positive constant $C_{0}$. Namely, for a given $t \in[0, T]$ let us define

$$
\varphi^{n}(x, s):=g^{n}\left(y+s D \rho_{-}(\nu(y)), t\right)-g\left(y+s D \rho_{-}(\nu(y)), t\right),
$$

for $x \in \Omega \backslash \bar{\Sigma}, \Pi(x)=\{y\}$, and $s \in\left[0, l^{-}(y)\right]$. We remark that, if $x \in \Omega \backslash \bar{\Sigma}$ and $y$ is the projection of $x$, then $\varphi^{n}(x, \cdot)=\varphi^{n}(y, \cdot)$.

By the very definition of $w^{n}, w$ and $M_{x}$, Hölder's inequality and (32) we have that

$$
\left\|w^{n}(t)-w(t)\right\|_{L^{p}(\Omega)}^{p} \leq C \int_{\Omega}\left(\int_{d_{\Omega}^{-}(x)}^{l^{-}(x)}\left|\varphi^{n}(x, s)\right|^{p} \prod_{i=1}^{n-1} \frac{1-s \tilde{\kappa}_{i}^{-}(x)}{1-d_{\Omega}^{-}(x) \tilde{\kappa}_{i}^{-}(x)} d s\right) d x=: I
$$


where $l^{-}(x):=l^{-}(y)$ when $\Pi^{-}(x)=\{y\}$ and $C:=M_{0}^{p-1}\left(\max d_{\Omega}^{-}\right)^{1 / p^{\prime}}$. Using the change of variables theorem (see [14, Thm. 7.1]) we have that

$$
\begin{aligned}
I= & C \int_{\partial \Omega} \rho_{-}(\nu(y))\left[\int_{0}^{l^{-}(y)}\left(\int_{\sigma}^{l^{-}(y)}\left|\varphi^{n}(y, s)\right|^{p} \prod_{i=1}^{n-1} \frac{1-s \tilde{\kappa}_{i}^{-}(y)}{1-\sigma \tilde{\kappa}_{i}^{-}(y)} d s\right)\right. \\
& \left.\cdot \prod_{i=1}^{n-1}\left(1-\sigma \tilde{\kappa}_{i}^{-}(y)\right) d \sigma\right] d \mathcal{H}^{n-1}(y) \\
= & C \int_{\partial \Omega} \rho_{-}(\nu(y))\left[\int_{0}^{l^{-}(y)}\left(\int_{\sigma}^{l^{-}(y)}\left|\varphi^{n}(y, s)\right|^{p} \prod_{i=1}^{n-1}\left(1-s \tilde{\kappa}_{i}^{-}(y)\right) d s\right) d \sigma\right] d \mathcal{H}^{n-1}(y) \\
\leq & C^{\prime} \int_{\partial \Omega} \rho_{-}(\nu(y))\left(\int_{0}^{l^{-}(y)}\left|\varphi^{n}(y, s)\right|^{p} \prod_{i=1}^{n-1}\left(1-s \tilde{\kappa}_{i}^{-}(y)\right) d s\right) d \mathcal{H}^{n-1}(y),
\end{aligned}
$$

where $C^{\prime}:=C \max d_{\Omega}^{-}$. Using again the change of variables theorem we finally get

$$
I \leq C^{\prime} \int_{\Omega}\left|g^{n}(x, t)-g(x, t)\right|^{p} d x
$$

hence $(50)$ follows.

Remark 8.5. Notice that if $d_{\Omega}^{-}(x)>\lambda_{i}^{n}(y)$, then $v_{i}^{n}(x)=0$, while $h_{i}^{n}(x)=c_{i}^{n}-d_{\Omega}^{-}(x)$ if $d_{\Omega}^{-}(x) \leq \lambda_{i}^{n}(y)$. Hence we have that $w^{n}(x, t) D \rho\left(D h^{n}(x, t)\right)=w^{n}(x, t) D \rho\left(-D d_{\Omega}^{-}(x)\right)$ a.e. in $\Omega$ and for every $t \in[0, T]$. A passage to the limit in (44) leads to

$$
-\operatorname{div}(w(x, t) D \rho(D h(x, t)))=-\frac{\partial H_{s}}{\partial t} \chi_{\left\{h_{0} \leq H_{s}(t)-d_{\Omega}^{-}\right\}},
$$

in the sense of distributions in $\Omega$ for every $t \in[0, T]$. Recalling that, by construction, $\frac{\partial H_{s}}{\partial t} \chi_{\left\{h_{0} \leq H_{s}(t)-d_{\Omega}^{-}\right\}}=\frac{\partial h}{\partial t}(x, t)$, and comparing (51) with the Faraday's law, we conclude that the electric field induced by $h$ inside the superconductor is $\vec{E}(x, t)=$ $w(x, t) D \rho(D h(x, t))$.

\section{Further REMARKS}

As a consequence of the Theorem 8.1 we obtain some detailed information about the macroscopic behavior of the penetrating magnetic field.

Saturation time (Full penetration time). The experiments show that, if the superconductor is posed in an increasing external field $H_{s}$, say $H_{s}(t) \rightarrow+\infty$ as $t \rightarrow+\infty$, after a while the internal magnetic field $h(x, t)$ differs from $H_{s}(t)$ for a stationary amount $u(x)$. In our model we obtain that if $\tau \geq 0$ is such that $H_{s}(\tau)=\sup _{\Omega}\left(h_{0}+d_{\Omega}^{-}\right)$, then for $t \geq \tau$ $h(x, t)-H_{s}(t)=-d_{\Omega}^{-}(x)$.

In the simpler case when $H_{s}(t)=-a t, a>0$, and $h_{0}=0$ we obtain that at time $\tau=\frac{\max _{\Omega} d_{\Omega}}{a}$ the magnetic field is penetrated in the whole superconductor and $h(x, t)=$ $d_{\Omega}(x)+H_{s}(t)$ for $t \geq \tau$. Figures 1 and 2 picture the plots of the magnetic field and of the dissipation in the stationary configuration for anisotropic materials with a complicated geometry. We underline that the numerical computation based on the representation formula (49) shows the presence of an hight dissipation near the points of the boundary with negative curvature, according to the experimental observations. 

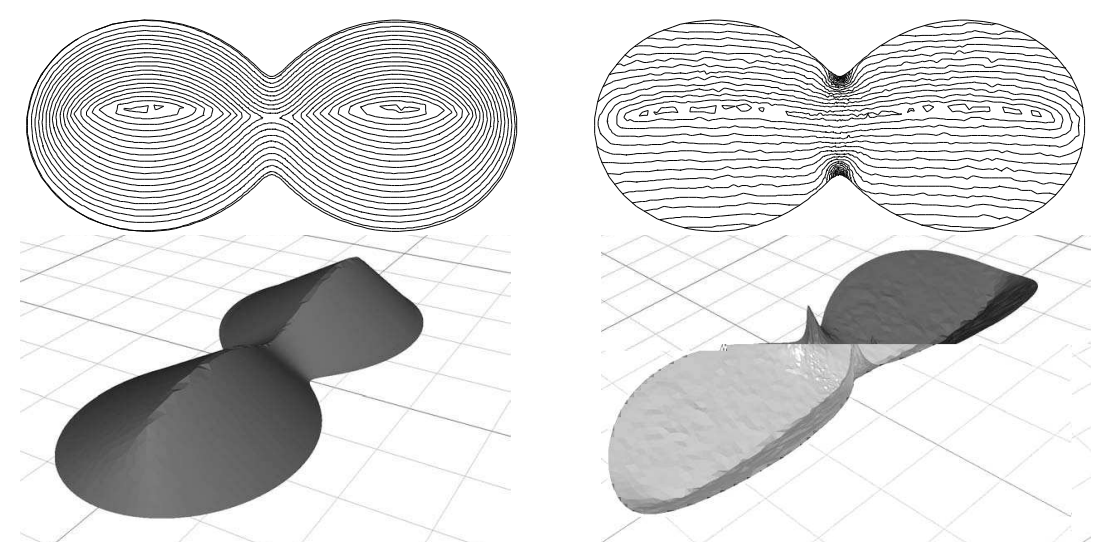

FiguRE 1. Level sets and 3D plot of the full penetrated magnetic field and of the dissipation for $\Omega$ Cassini's Egg, $K$ ellipse not centered at the origin, $h_{0}=0$
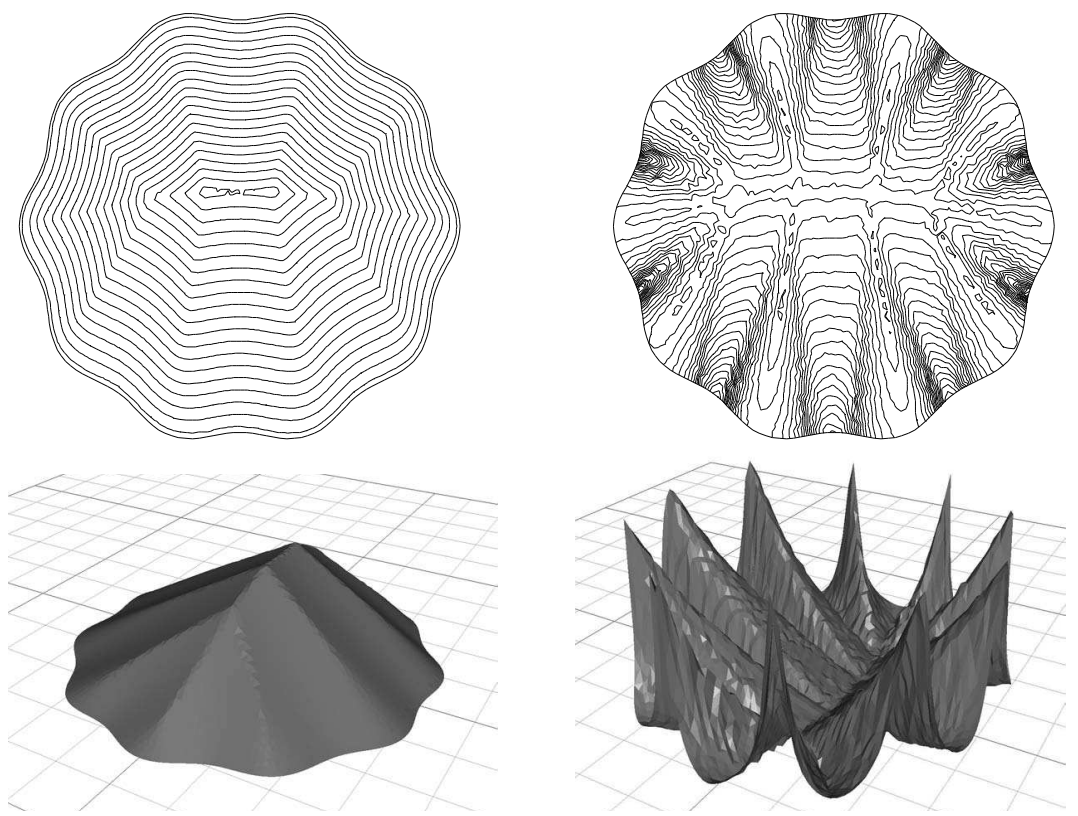

FiguRE 2. Level sets and 3D plot of the full penetrated magnetic field and of the dissipation for $\Omega$ perturbed disk, $K$ ellipse not centered at the origin, $h_{0}=0$.

Boundary of penetration profile. If the evolution starts with $h_{0}=0$ (no initial magnetic field inside $\Omega$ ) and the external field is nondecreasing, then at time $t$ the induced magnetic field is penetrated in $\Omega$ only in the region $\left\{x \in \Omega: d_{\Omega}^{-}(x) \leq H_{s}(t)\right\}$. This gives an exact macroscopic description of the boundary of penetration profile, which is given by the level sets of the Minkowski distance from the boundary of $\Omega$.

Hysteresis phenomenon. Assume that the evolution starts with $h_{0}=0$ (no initial magnetic field inside $\Omega$ ) and that the external field $H_{s}(t)$ is increasing in $[0, T]$, then at time $T$ the induced magnetic field is $h(x, T)=\left(-d_{\Omega}^{-}(x)+H_{s}(t)\right)_{+}$. Starting from this configuration, 

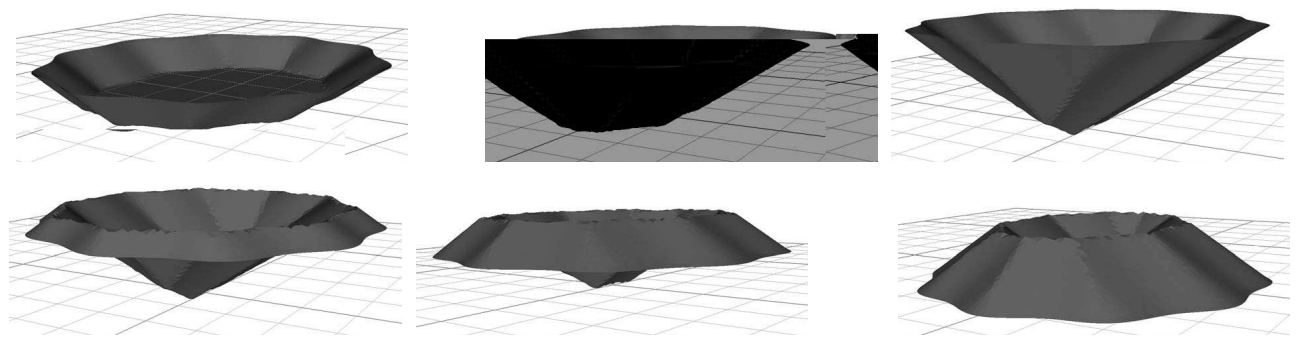

Figure $3 . \Omega$ perturbed disk, $K$ ellipse centered at the origin. Profile of the internal magnetic field, starting from $h_{0}=0$, during a loop of the external field.

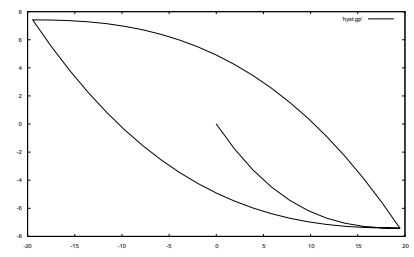

FiguRE 4. Magnetic hysteresis loop corresponding to the previous process.

in [T,2T] the superconductor is subject to the external field $H_{s}(2 T-t)$. Then we have $h(x, 2 T)=\min \left\{\left(-d_{\Omega}^{-}(x)+H_{s}(T)\right)_{+}, d_{\Omega}(x)+H_{s}(0)\right\}$ which in general is not zero. This corresponds with the experimental observation of an hysteresis phenomenon for these hard superconductors after an external field loop.

On the validity of the Badiá-López formalism for anisotropic materials. In 17 Fisher doubts the validity of the variational formulation proposed by Badía and Lopez in the case of anisotropic materials. He declares that "one cannot be sure that the suggested least action principle chooses the Current-Voltage Characteristics correctly. Of course, if this principle is proved, it would be a very important tool to determine the Current-Voltage Characteristics via the known restriction region". Our results state that, if the powerlaw approximation for the dissipation is assumed to be reliable, then the Current-Voltage Characteristics induced by this approximation is the one selected by the minimization procedure.

\section{REFERENCES}

[1] A. Badía and C. López, Vector magnetic hysteresis of hard superconductors, Phys. Rev. B 65 (2001), 104514.

[2] A. Badía and C. López, Horizons in Superconductivity Research, ch. Minimal model for the topology of the critical state in hard superconductors, Nova Science Publishers, 2003.

[3] A. Badía and C. López, Electric field in hard superconductors with arbitrary cross section and general critical current law, J. Appl. Phys. 95 (2004), 8035-8040.

[4] M. Bardi and I. Capuzzo Dolcetta, Optimal Control and Viscosity Solutions of Hamilton-JacobiBellman Equations, Systems \& Control: Foundations \& Applications, Birkhäuser, Boston, 1997.

[5] J.W. Barrett and L. Prigozhin, Sandpiles and superconductors: dual variational formulations for critical-state problems, preprint, 2005.

[6] J.W. Barrett and L. Prigozhin, Bean's critical-state model as the $p \rightarrow \infty$ limit of an evolutionary p-Laplacian equation, Nonlinear Anal. 42 (2000), 977-993.

[7] C.P. Bean, Magnetization of hard superconductors, Phys. Rev. Letters 8 (1962), 250-253. 
[8] K.V. Bhagwat, D. Karmakar, and G. Ravikumar, Critical state model with anisotropic critical current density, J. Phys.: Condens. Matter 15 (2003), 1325-1337.

[9] A. Braides, $\Gamma$-convergence for beginners, Oxford University Press, New York, 2002.

[10] E.H. Brandt, Electric field in superconductors with rectangular cross section, Phys. Rev. B 52 (1995), $15442-15457$.

[11] G. Buttazzo, Semicontinuity, relaxation and integral representation in the calculus of variations, Pitman Res. Notes Math. Ser., vol. 207, Longman Scientific and Technical, Harlow, U.K., 1989.

[12] P. Cannarsa and C. Sinestrari, Semiconcave functions, Hamilton-Jacobi equations and optimal control, Progress in Nonlinear Differential Equations and their Applications, vol. 58, Birkhäuser, Boston, 2004.

[13] S.J. Chapman, A Hierarchy of Models for Type-II Superconductors, SIAM Rev. 42 (2000), 555-598.

[14] G. Crasta and A. Malusa, The distance function from the boundary in a Minkowski space, to appear in Trans. Amer. Math. Soc., oai:arXiv:math.AP/0612226.

[15] G. Crasta and A. Malusa, On a system of partial differential equations of Monge-Kantorovich type, to appear in J. Differential Equations, oai:arXiv:math.AP/0612227.

[16] G. Dal Maso, An introduction to $\Gamma$-convergence, Birkhäuser, Boston, 1993.

[17] L.M. Fisher and V.A. Yampol'skii, Comment on "Critical statetheory for non-parallel flux line lattices in type-II superconductors", oai:arXiv:cond-mat/0201286, 2002.

[18] A. Garroni, V. Nesi, and M. Ponsiglione, Dielectric breakdown: optimal bounds, Proc. R. Soc. Lond. A 457 (2001), 2317-2335.

[19] S.I. Goldberg, Curvature and homology, Academic Press, New York, 1970.

[20] Y.Y. Li and L. Nirenberg, The distance function to the boundary, Finsler geometry and the singular set of viscosity solutions of some Hamilton-Jacobi equations, Commun. Pure Appl. Math. 58 (2005), 85-146.

[21] P.L. Lions, Generalized solutions of Hamilton-Jacobi equations, Pitman, Boston, 1982.

[22] R.T. Rockafellar, Convex Analysis, Princeton Univ. Press, Princeton, NJ, 1970.

[23] R. Schneider, Convex bodies: the Brunn-Minkowski theory, Cambridge Univ. Press, Cambridge, 1993.

[24] W. von Wahl, Estimating $\nabla u$ by divu and curlu, Math. Methods Appl. Sci. 15 (1992), 123-143.

[25] H.-M. Yin, B.Q. Li, and J. Zou, A degenerate evolution system modeling Bean's critical-state type-II superconductors, Discrete Contin. Dynam. Systems 8 (2002), 781-794.

Dipartimento di Matematica "G. Castelnuovo", Univ. di Roma I, P.le A. Moro 2 - 00185 Roma (ITALy)

E-mail address, Graziano Crasta: crasta@mat.uniroma1.it

E-mail address, Annalisa Malusa: malusa@mat.uniroma1.it 